\title{
Entrainment and Mixing in Stratocumulus: Effects of a New Explicit Subgrid-Scale Scheme for Large-Eddy Simulations with Particle-Based Microphysics
}

\author{
FABIAN HOFFMANN \\ Cooperative Institute for Research in Environmental Sciences, University of Colorado Boulder, and \\ NOAA/Earth System Research Laboratory/Chemical Sciences Division, Boulder, Colorado \\ GRAHAM FEINGOLD \\ NOAA/Earth System Research Laboratory/Chemical Sciences Division, Boulder, Colorado
}

(Manuscript received 22 October 2018, in final form 30 March 2019)

\begin{abstract}
The entrainment and mixing of free-tropospheric air is an essential component of the observed microphysical structure of stratocumulus clouds. Since the relevant scales involved in this process are usually smaller than the grid spacing of typical large-eddy simulations (LESs), their correct representation is difficult. To adequately accommodate these small-scale processes, we apply a recently developed approach that explicitly simulates LES subgrid-scale (SGS) turbulence fluctuation of supersaturation using the onedimensional linear eddy model. As a result of reduced numerical diffusion and the ability to explicitly represent the SGS distribution of liquid water and supersaturation, entrainment rates tend to be lower in the new approach compared to simulations without it. Furthermore, cloud holes comprising free-tropospheric air with negligible liquid water are shown to persist longer in the stratocumulus deck. Their mixing with the cloud is shown to be more sensitive to the microphysical composition of the cloud as a result of the explicitly resolved inhomogeneous mixing, which is also confirmed analytically. Moreover, inhomogeneous mixing is shown to decrease the droplet concentration and to increase droplet growth significantly, in contrast to previous studies. All in all, the simulations presented can be seen as a first step to bridge the gap between ultrahigh-resolution direct numerical simulation and LES, allowing an appropriate representation of small-scale mixing processes, together with the large-scale dynamics of a stratocumulus system.
\end{abstract}

\section{Introduction}

Stratocumulus are the most abundant cloud type, covering about one-fifth of Earth's surface. They have a strong negative (i.e., cooling) impact on Earth's radiation budget by increasing the reflection of incident shortwave radiation while having almost no effect on the emission of longwave radiation. Despite their ubiquity and importance for the climate system, our ability to represent them in numerical models is limited. The processes of entrainment and mixing are especially challenging even in highresolution large-eddy simulations (LESs).

Entrainment and mixing introduce free-tropospheric air into the cloud and hence the stratocumulus-topped boundary layer, where they increase temperature, decrease

Corresponding author: Fabian Hoffmann, fabian.hoffmann@ noaa.gov humidity, and change the microphysical composition of the cloud. Accordingly, understanding entrainment and mixing is necessary to understand whether a stratocumulus deck is maintained or dissipates (Lilly 1968), to assess its influence on the radiation budget (Jeffery 2007), or to estimate its ability to precipitate (Baker et al. 1980). In fact, Magaritz-Ronen et al. (2014) have shown that in the absence of entrainment and mixing, simulations do not reproduce the microphysical and thermodynamical characteristics of observed stratocumulus.

Entrainment in stratocumulus starts in the so-called entrainment interface layer, a transition region between the cloud and free troposphere, in which free-tropospheric air is cooled and moistened by a combination evaporation of detrained cloudy air and the emission of longwave radiation (e.g., Wood 2012; Yamaguchi and Randall 2012; Mellado 2017). From the entrainment interface layer, sufficiently cooled volumes of negligible or depleted liquid 
water, so-called cloud holes, descend slowly into the cloud layer (Nicholls 1989; Gerber et al. 2005). Here, they continue to sink deeper, either because of further evaporative cooling as a result of the ongoing mixing with the cloud, as hypothesized by Gerber et al. (2005) and Haman (2009), or by following the subsiding branches of stratocumulus large-eddy circulation (Yamaguchi and Randall 2012), until they homogenize with the cloud. Besides the aforementioned effects of dynamics, thermodynamics, and radiation, the microphysical composition of a cloud, that is, the number and size of cloud droplets, not only modifies entrainment and mixing but is itself changed by the entrainment and mixing processes.

Cloud microphysics modify entrainment and mixing in two ways: (i) the sedimentation-entrainment feedback (Ackerman et al. 2004; Bretherton et al. 2007), in which larger droplets remove liquid water from the cloud interface more effectively, decreasing the potential for evaporative cooling and hence slowing down turbulent mixing, and (ii) the evaporation-entrainment feedback (Wang et al. 2003), in which a larger total droplet surface area accelerates evaporation and hence increases turbulent mixing. Together, both feedbacks result in a higher entrainment rate in clouds with larger droplet concentrations (assuming a constant liquid water content). Both feedbacks can be considered as manifestations of the cloud-top entrainment instability without the traditionally required, but usually not observed, complete dissipation of the cloud (Lilly 1968; Yamaguchi and Randall 2008).

Cloud microphysics are changed by turbulent mixing as a result of the interplay of turbulence and commensurate microphysical reaction by evaporation (Baker and Latham 1979; Baker et al. 1980; Feingold and Siebert 2009). The character of mixing is quantified in terms of the Damköhler number,

$$
\mathrm{Da}=\frac{\tau_{\text {mix }}}{\tau_{\text {react }}},
$$

where $\tau_{\text {mix }}=\left(l^{2} / \varepsilon\right)^{1 / 3}$ measures the time required for an eddy of length $l$ to break down to the Kolmogorov length scale at a turbulence kinetic energy dissipation rate of $\varepsilon$, and $\tau_{\text {react }}$ is a time scale associated with the evaporation process, usually the phase relaxation time scale that measures the time needed to saturate a subsaturated volume of air by evaporation (Squires 1952):

$$
\tau_{\text {phase }} \approx\left(4 \pi D_{v} N r_{m}\right)^{-1},
$$

where $D_{v}$ is the molecular diffusion coefficient for water vapor in air, $N$ the droplet number concentration, and $r_{m}$ the arithmetic-mean droplet radius. Based on Da, mixing can be bounded by homogeneous mixing, where faster turbulence produces a uniform subsaturation causing partial evaporation of all droplets $(\mathrm{Da} \ll 1)$, and (extreme) inhomogeneous mixing, where turbulence is so ineffective at mixing that bodies of cloudy and cloudfree air remain effectively disjunct, and only those droplets that are moved outside the cloud evaporate completely ( $\mathrm{Da} \gg 1$ ). Therefore, homogeneous mixing maintains the droplet concentration but decreases the mean drop size, while (extreme) inhomogeneous mixing maintains the mean drop size but decreases the droplet concentration. Together, homogeneous and extreme inhomogeneous mixing frame the potential impacts of mixing on cloud microphysics. The actual changes due to homogeneous and inhomogeneous mixing, however, also depended on the initial subsaturation of the cloudfree air (Pinsky et al. 2016).

In principle, all these processes can be resolved in LES. However, LES is well known to overestimate entrainment in stratocumulus (Stevens et al. 2005). The reason is twofold: First, essential underlying dynamical and microphysical processes are associated with length scales far smaller than the typical resolution of today's LES (on the order of tens of meters), and the parameterization of the LES subgrid-scale (SGS) turbulence in analogy to molecular diffusion is inadequate at those resolutions (Mellado et al. 2018). These restrictions also force the SGS mixing to be homogeneous, although it is known to be inhomogeneous on scales larger than just a couple of centimeters to decimeters depending on turbulence and microphysical composition (Lehmann et al. 2009). Second, numerical diffusion tends to weaken the typically strong gradients between air originating from the cloud and the free troposphere spuriously, accelerating the exchange between those bodies (e.g., Stevens and Bretherton 1999), or smearing out small-scale dynamical features of the entrainment process like cloud holes. Of course, these pitfalls can be avoided by direct numerical simulation (DNS), in which dynamics are resolved down to the smallest relevant length scale, the Kolmogorov length scale. Because of the enormous computational costs, however, these simulations are restricted to very limited computational domains. Nonetheless, to investigate large domains, LES can be tuned by the ratio of horizontal to vertical grid spacing to obtain a realistic entrainment rate (Pedersen et al. 2016), and SGS mixing can be adapted to parameterize the desired scenario of homogeneous to inhomogeneous mixing by altering the microphysical variables commensurately (Hill et al. 2009; Jarecka et al. 2009).

Another approach to overcome these issues is to use a more suitable LES SGS model, as recently developed 
by the authors (Hoffmann et al. 2019). The new modeling approach combines LES with the so-called linear eddy model (LEM) of Kerstein (1988) and a Lagrangian cloud model (LCM) [LES-LEM-LCM (L3)]. The LEM represents LES SGS turbulence and mixing explicitly by representing turbulent compression and folding, as well as molecular diffusion (in principle) down to the Kolmogorov length scale in a computationally efficient one-dimensional domain (e.g., Krueger 1993; Krueger et al. 1997; Su et al. 1998). The LCM is used to track the motion of air parcels between the LEMs of different grid boxes, in addition to its more typical application to represent cloud microphysics (e.g., Andrejczuk et al. 2008; Shima et al. 2009; Riechelmann et al. 2012; Hoffmann et al. 2015; Arabas et al. 2015; Grabowski et al. 2018). Moreover, while LCMs avoid the numerical diffusion of liquid water (e.g., Sato et al. 2018), L3 also prevents the numerical diffusion of supersaturation. In so doing, Hoffmann et al. (2019) showed that this approach is able to represent the microphysical composition of clouds under inhomogeneous mixing realistically, a process that is also frequently observed in stratocumulus (e.g., Pawlowska et al. 2000; Gerber et al. 2005).

In this study, effects of the new L3 modeling approach on the representation of entrainment and mixing in stratocumulus will be investigated. The analysis will cover (i) effects on cloud microphysics and how these change because of the improved representation of inhomogeneous mixing; (ii) the representation of smallscale dynamical features of the entrainment process, namely cloud holes, and how they mix with the cloud; and (iii) how the drop concentration affects the entrainment and mixing processes. The paper is organized as follows. Section 2 will give a brief overview of the L3 modeling approach and simulation setups. Section 3 will present the modeling results, focusing on the general properties of the stratocumulus, changes in microphysics, and effects on entrainment dynamics. The paper is concluded in section 4. An appendix will elaborate on the treatment of sedimentation in L3.

\section{Modeling framework and setup}

The dynamical core of L3 is the nonhydrostatic, anelastic LES model System for Atmospheric Modeling (SAM) by Khairoutdinov and Randall (2003), which predicts the three velocity components, liquid water static energy, water vapor mixing ratio, and SGS turbulent kinetic energy, which is used in SAM's 1.5thorder SGS scheme (Deardorff 1980). SAM is two-way coupled with the LCM, simulating cloud microphysics and the effects of explicit SGS turbulence. The LCM's main components are adumbrated below, and the reader is referred to Hoffmann et al. (2019) for a thorough description of the new L3 modeling approach and to Hoffmann et al. $(2015,2017)$ and Hoffmann (2017) for the general formulation of the LCM.

The LCM uses so-called superdroplets, each representing an ensemble of identical real droplets, to represent cloud microphysics. Each superdroplet has certain properties, which depict, inter alia, the number of droplets represented by each superdroplet (the socalled weighting factor), the radius of these droplets, the superdroplet's location in space, its velocity, and a perturbation of the absolute supersaturation $\delta_{n}^{\prime}$. The aforementioned property $\delta_{n}^{\prime}$ characterizes a virtual volume of air surrounding each superdroplet, used to consider thermodynamic and microphysical effects of the explicitly resolved SGS turbulence calculated by the LEM. The value of $\delta_{n}^{\prime}$ is determined for each superdroplet individually, following its trajectory through the LES domain, and is calculated to counteract the homogeneous mixing calculated in the LES SGS model and other numerical errors of the LES such as numerical diffusion of the supersaturation. The reader is referred to appendix A of Hoffmann et al. (2019) for more information on the determination of $\delta_{n}^{\prime}$.

The LEM is used to redistribute $\delta_{n}^{\prime}$ among all superdroplets in an LES grid box in accordance with the degree of SGS turbulence. This is done by arranging all values of $\delta_{n}^{\prime}$ into a one-dimensional array based on each superdroplet's origin, as detailed in Hoffmann et al. (2019). From this array, segments are chosen randomly to undergo the so-called triplet map that steepens gradients in a specific way, mimicking compression and folding of turbulent mixing (e.g., Kerstein 1988; Krueger 1993; Hoffmann et al. 2019). Finally, molecular diffusion is applied to the entire LEM. Since the physical length of the LEM matches the LES vertical grid spacing, the LEM resolution is given by the ratio of the vertical LES grid spacing to the number of superdroplets per grid box. Although this resolution is usually much higher than that of the LES, the LEM resolution does not necessarily capture all scales down to the physical Kolmogorov length scale. Therefore, molecular diffusion is replaced by adequately scaled turbulent diffusion to represent the effects of turbulent mixing on scales unresolved in the LEM (Krueger et al. 1997).

The perturbation absolute supersaturation $\delta_{n}^{\prime}$ is used alongside the LES-resolved absolute supersaturation $\bar{\delta}=\overline{q_{v}}-q_{s}(\bar{T}, p)$, where $\overline{q_{v}}$ is the LES water vapor mixing ratio and $q_{s}(\bar{T}, p)$ the saturation vapor mixing ratio calculated from the LES absolute temperature $\bar{T}$ and hydrostatic pressure $p$, to calculate the diffusional growth of a superdroplet by 


$$
\left(r_{n}+r_{0}\right) \frac{d r_{n}}{d t}=G\left(\bar{\delta}+\delta_{n}^{\prime}\right) f_{v}\left(r_{n}\right),
$$

where $r_{n}$ is the radius of the droplets represented by superdroplet $n, r_{0} \approx 1.86 \mu \mathrm{m}$ a length scale associated with gas kinetic effects (Mordy 1959; Kogan 1991), $G=\left[q_{s}(\bar{T}, p)\left(F_{d}+F_{k}\right)\right]^{-1}$ summarizes the effects of vapor diffusion and heat conduction on condensation [for definitions of $F_{d}$ and $F_{k}$, see Rogers and Yau (1989, p. 102)], and $f_{v}\left(r_{n}\right)$ is a factor parameterizing ventilation effects on diffusional growth depending on the droplet radius (Rogers and Yau 1989, p. 116). Note that the use of a perturbation supersaturation alongside the LESresolved supersaturation in (3) is similar to the approach developed by Grabowski and Abade (2017) and Abade et al. (2018), who determine the perturbation supersaturation in a fundamentally different way based on the stochastic fluctuations of the vertical velocity. Note further that the initial particle radius for integrating (3) is set to $0.01 \mu \mathrm{m}$, and particles are not allowed to evaporate below this size. Particles with radii larger than $1.0 \mu \mathrm{m}$ are considered as cloud droplets since activation is not considered explicitly in this study. This simplification, however, allows an analytical solution of the stiff differential equations, (3), and does not require very short integration time steps at small droplet radii that would be required if surface tension and solute effects were to be included. Furthermore, collision and coalescence are neglected here.

The motion of a superdroplet is described by

$$
\frac{d X_{i, n}}{d t}=\bar{u}_{i}+u_{i, n}^{\prime}(\bar{e})-\delta_{i 3} w_{s}\left(r_{n}\right),
$$

where $X_{i, n}$ is the location of the $n$th superdroplet in Cartesian coordinates $\left(X_{i}\right)=(X, Y, Z),\left(\bar{u}_{i}\right)=(\bar{u}, \bar{v}, \bar{w})$ is the LES-resolved velocity interpolated to the superdroplet's location, $\left(u_{i, n}^{\prime}\right)=\left(u_{n}^{\prime}, v_{n}^{\prime}, w_{n}^{\prime}\right)$ is an autocorrelated stochastic velocity component calculated from the SGS turbulence kinetic energy $\bar{e}$ (Sölch and Kärcher 2010), $\delta_{i 3}$ is the Kronecker delta, and $w_{s}$ is the sedimentation velocity depending (primarily) on the droplet radius $r_{n}$ (Beard 1976). In contrast to the description of L3 in Hoffmann et al. (2019), droplet sedimentation is considered here. Since L3 does not inherently allow sedimentation, L3 is only applied to those superdroplets for which sedimentation is negligible in comparison with their turbulent motion. If, however, a superdroplet sediments significantly, this superdroplet is neglected in the calculations of the LEM, and it experiences standard homogeneous mixing $\left(\delta^{\prime}{ }_{n}=0\right)$. For those superdroplets sedimenting insignificantly, this procedure results in a slight underestimation of mixing in the LEM (see also Tölle and Krueger 2014). Estimates of this underestimation and more details on the consideration of sedimentation are given in the appendix.

All simulations in this study are based on the LES intercomparison case derived from the first research flight (RF01) of the Second Dynamics and Chemistry of the Marine Stratocumulus field study (DYCOMS II) (Stevens et al. 2003). The case represents a maritime nocturnal stratocumulus deck and is described by Stevens et al. (2005) in more detail. All simulations use a constant vertical grid spacing of $5 \mathrm{~m}$ and a horizontal grid spacing of $35 \mathrm{~m}$. In total, 288 grid boxes are simulated in the vertical and 96 in each horizontal direction. In each grid box, 100 superdroplets are initialized. The superdroplet weighting factors are the same for all superdroplets in a single simulation, but are adapted for different simulations to represent droplet number concentrations of 100,200 , and $400 \mathrm{~cm}^{-3}$, which will be represented by blue, green, and red lines, respectively. L3 simulations will be addressed as LEM simulations, since the usage of the LEM is the main difference from the control simulations in which the LEM is turned off (i.e., $\delta_{n}^{\prime}=0$ ). Accordingly, the control simulations treat the SGS supersaturation homogeneously and are therefore addressed as homogeneous simulations. The homogeneous simulations will be depicted in pale colors matching those of the corresponding LEM simulations. The grid spacing of the LEM is approximately $5 \mathrm{~cm}$, resolving the entire range of inhomogeneous mixing, which becomes homogeneous below a length scale of about (Lehmann et al. 2009)

$$
l_{t}=\varepsilon^{1 / 2} \tau_{\text {react }}^{3 / 2} \approx 8 \mathrm{~cm},
$$

where $\varepsilon \approx 10 \mathrm{~cm}^{2} \mathrm{~s}^{-3}$ and $\tau_{\text {react }}=\tau_{\text {phase }} \approx 1.8 \mathrm{~s}$ (using values derived from the $400-\mathrm{cm}^{-3}$ simulation, which would increase for simulations with smaller droplet number concentrations). All simulations are computed with a time step of $0.5 \mathrm{~s}$; the LEM is subcycled if necessary. Analysis is restricted to the last two hours of the 4-h simulations.

\section{Results}

First, we focus on general properties of the analyzed stratocumulus deck before advancing to distinct changes in cloud microphysics (section 3a) and entrainment-related dynamics of cloud holes (section 3b).

Figure 1 shows the cloud water mixing ratio $q_{c}$ (Fig. 1a), fraction of cloud droplets $\left(N_{c} / N_{\text {init }}\right)$ (Fig. 1b), mean SGS perturbation supersaturation $\mu_{S^{\prime}}$ (Fig. 1c), and standard deviation of the SGS perturbation supersaturation $\sigma_{S^{\prime}}$ (Fig. 1d) as vertical cross sections (left column) and horizontally and temporally (last $2 \mathrm{~h}$ of the 

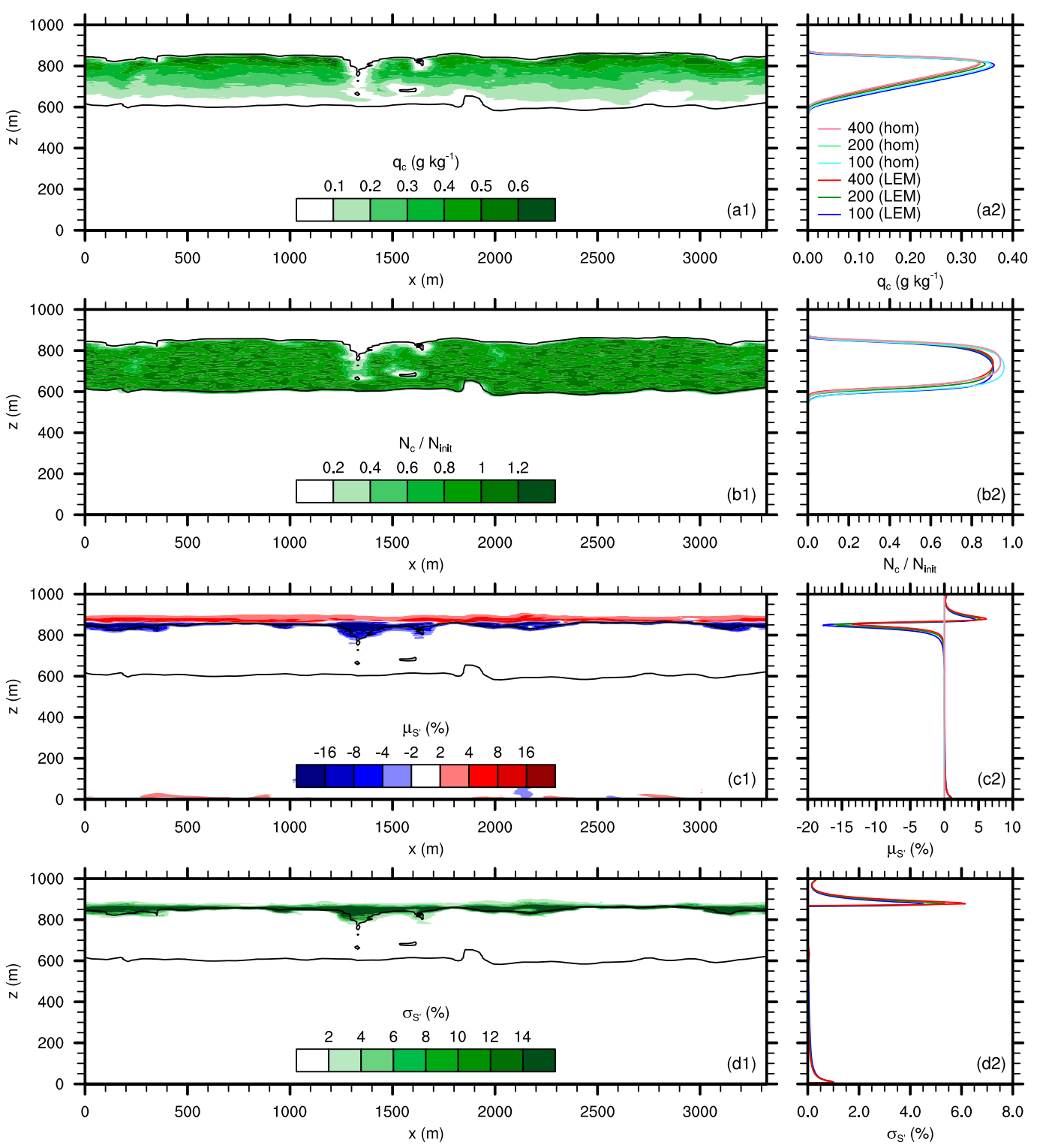

FIG. 1. (left) Vertical cross sections and (right) profiles of (a) the cloud water mixing ratio, (b) fraction of cloud droplets $\left(N_{c} / N_{\text {init }}\right)$, (c) mean value of SGS supersaturation perturbation $\mu_{S^{\prime}}$, and (d) standard deviation of SGS supersaturation perturbation $\sigma_{S^{\prime}}$. The cross sections show results from a simulation with $N_{\text {init }}=200 \mathrm{~cm}^{-3}$ and the LEM as an SGS model. The profiles show results for initial droplet concentrations of $N_{\text {init }}=100,200$, and $400 \mathrm{~cm}^{-3}$ (blue, green, and red, respectively) and for simulations using the LEM as an SGS model (LEM, saturated colors) or assuming homogeneity on the subgrid scale (hom, pale colors).

simulation) averaged profiles (right column). The latter two quantities are defined as

$$
\mu_{S^{\prime}}=\frac{1}{N} \sum_{n=1}^{N} \delta_{n}^{\prime} / q_{s}(\bar{T}, p)
$$

and

$$
\sigma_{S^{\prime}}=\sqrt{\frac{1}{N-1} \sum_{n=1}^{N}\left[\delta_{n}^{\prime} / q_{s}(\bar{T}, p)-\mu_{S^{\prime}}\right]^{2}},
$$

where $N$ denotes the number of superdroplets per grid box. The cross sections are selected from the LEM simulation with an initial particle concentration of $N_{\text {init }}=200 \mathrm{~cm}^{-3}$. The profiles show results from LEM and homogeneous simulations with all investigated droplet number concentrations.

The cross sections and profiles of $q_{c}$ and $N_{c} / N_{\text {init }}$ exhibit canonical behavior; that is, $q_{c}$ increases almost linearly from cloud base and $N_{c} / N_{\text {init }}$ is almost constant throughout the cloud. The change in these quantities 

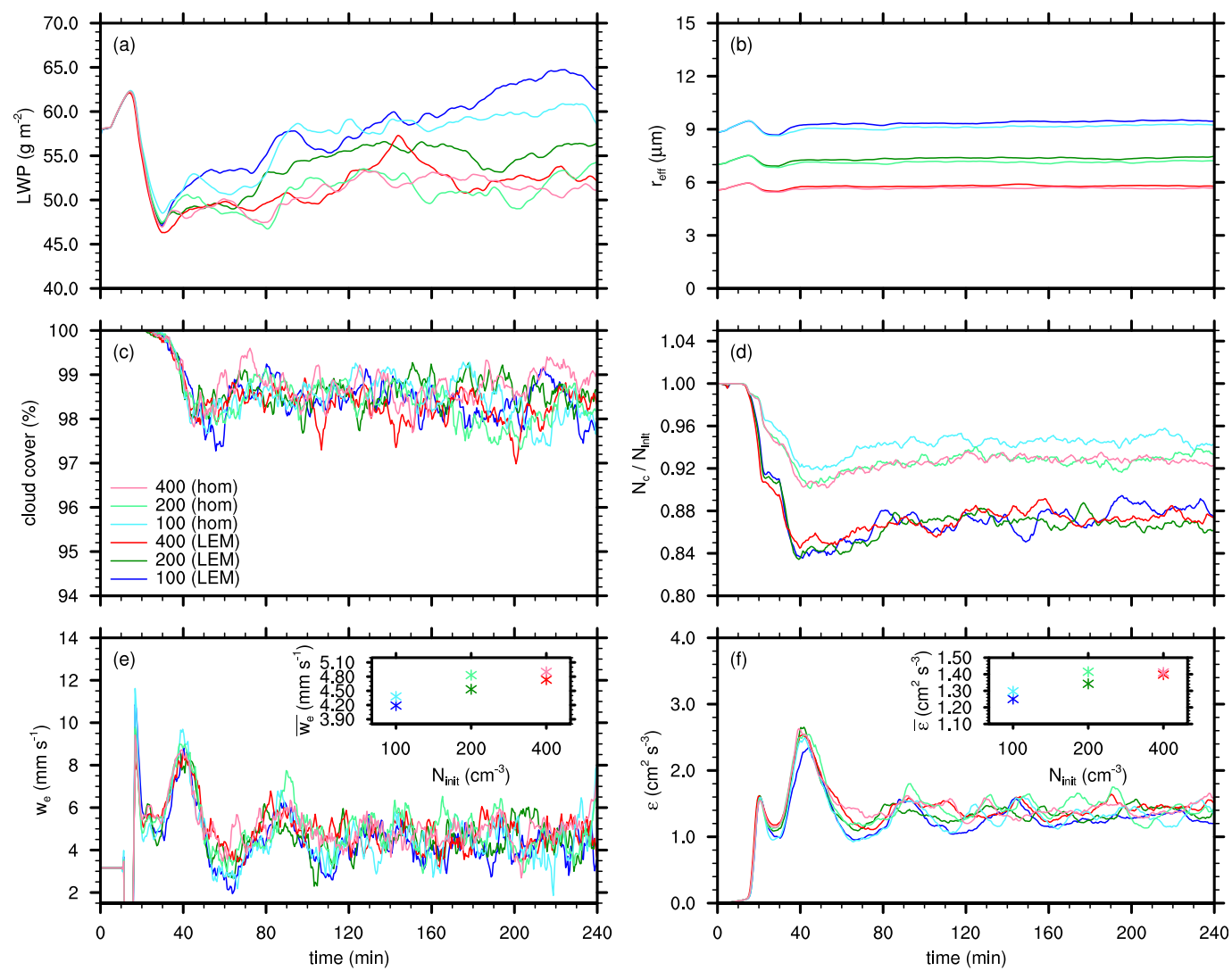

FIG. 2. Time series of (a) liquid water path, (b) mean effective radius, (c) cloud cover, (d) fraction of cloud droplets $\left(N_{c} / N_{\text {init }}\right)$, (e) entrainment velocity $w_{e}$, and (f) in-cloud dissipation rate $\varepsilon$. The insets in (e) and (f) show averages over the last $2 \mathrm{~h}$. See (c) for color code.

due to changes in $N_{\text {init }}$ is also well described: as a result of the evaporation- and sedimentation-entrainment feedbacks, the transport of free-tropospheric air into the boundary layer increases with increasing $N_{\text {init }}$ (see also the entrainment velocity in Fig. 2e), warming and drying the boundary layer, and eroding the cloud from its base (Wang et al. 2003).

The spatial distribution of $\mu_{S^{\prime}}$ and $\sigma_{S^{\prime}}$ exhibit their largest absolute values at the inversion, where $\mu_{S^{\prime}}$ also changes its sign. While $\sigma_{S^{\prime}}$ indicates the SGS mixing calculated in the LEM, the nonzero values for $\mu_{S^{\prime}}$ indicate the longer-lasting spatial redistribution of water and temperature due to the use of the LEM. Negative values of $\mu_{S^{\prime}}$ below the inversion indicate warm and dry free-tropospheric air that has been entrained into the boundary layer, while positive values above show cold and moist boundary layer air detrained into the free troposphere. This area of nonzero $\mu_{S^{\prime}}$ and $\sigma_{S^{\prime}}$ also marks the entrainment interface layer, in which air masses originating from the cloud layer and the free troposphere coexist, mix, and precondition the entrained air (e.g., Stevens et al. 2003; Yamaguchi and Randall 2012).
Additionally, one can see that nonzero values of $\mu_{S^{\prime}}$ and $\sigma_{S^{\prime}}$ extend into cloud-free entities below the average inversion height, with $\mu_{S^{\prime}}$ and $\sigma_{S^{\prime}}$ fading with increasing distance from the inversion. These so-called cloud holes are known to be preferred regions of the ultimate mixing between preconditioned free-tropospheric air and the cloud (e.g., Nicholls 1989; Gerber et al. 2005; Yamaguchi and Randall 2012; Yamaguchi and Feingold 2013), as also indicated by the reduced liquid water content and cloud droplet number concentration in their vicinity [Figs. $1 \mathrm{a}(1)$ and $1 \mathrm{~b}(1)$ ]. Cloud holes will be further analyzed in section $3 b$.

Generally, the magnitude of $\mu_{S^{\prime}}$ and $\sigma_{S^{\prime}}$ decreases with increasing $N_{\text {init }}$, which must be related to the increase of cloud turbulence (see also the kinetic energy dissipation rate in Fig. 2f) in more polluted clouds due to the evaporation- and sedimentation-entrainment feedbacks, overall driving a more homogeneous mixing process. This is interesting since both the numerator and the denominator of the Damköhler number [see (1)] decrease for higher droplet concentrations as a result of stronger turbulence and faster evaporation due to more 
numerous droplets, respectively. This complicates the prediction of the mixing type based to changes in the droplet concentration (Feingold and Siebert 2009). The results presented indicate, however, that the increase in turbulence outweighs the accelerated evaporation, leading to more homogeneous mixing.

Finally, note that comparably small nonnegative values of $\mu_{S^{\prime}}$ and $\sigma_{S^{\prime}}$ also occur close to the surface $(z<100 \mathrm{~m})$. The reason for this is the inability of the LES to resolve turbulence successfully close to an impermeable surface (Sagaut 2006, chapter 10), resulting in stronger contribution of the LES SGS model or, in this case, the LEM. Because of their distance to the cloud, they have no effect on the simulated cloud properties.

Figure 2 shows time series of the liquid water path (LWP) (Fig. 2a), mean effective radius $r_{\text {eff }}$ (Fig. 2b), cloud cover (Fig. 2c), fraction of cloud droplets $\left(N_{c} / N_{\text {init }}\right)$ (Fig. 2d), entrainment velocity $w_{e}$ (Fig. 2e), and in-cloud kinetic energy dissipation rate $\varepsilon$ (Fig. 2f) as determined in the LES SGS model. The cloud cover is calculated from the fraction of columns exceeding an optical thickness of 1 , and the entrainment velocity is determined as

$$
w_{e}=\frac{d z_{i}}{d t}-\langle w\rangle_{z_{i}}
$$

where $z_{i}$ is the domain-averaged inversion height, determined as the height of the maximum vertical gradient of the potential temperature in each column, and $\langle w\rangle_{z_{i}}$ is the average vertical fluid velocity at $z_{i}$, calculated from the large-scale divergence of the horizontal wind. The insets in Figs. 2e and 2f show the temporal mean of the corresponding quantities derived over the last two hours of each simulation.

The LWP and cloud cover time series are in agreement with the range of values reported by Stevens et al. (2005). The LWP shows a clear dependence on $N_{\text {init }}$, with higher values for low $N_{\text {init }}$, again in agreement with the evaporation- and the sedimentation-entrainment feedbacks. This is supported by the entrainment velocity in Fig. 2e, showing clearly that the entrainment velocity increases with $N_{\text {init }}$ (see inset). A similar trend can be seen in the dissipation rate (Fig. 2f), which increases as a result of stronger entrainment, which in turn initiates stronger evaporative cooling and hence a higher turbulent kinetic energy in the cloud.

By comparing the homogeneous simulations to simulations with the explicit consideration of SGS supersaturation fluctuations by the LEM, one can see a clear increase in the entrainment velocity for the homogenous simulations with commensurate changes in the LWP and dissipation rate. To show that the small differences in the insets of Figs. 2e and $2 \mathrm{f}$ are statistically significant,
Student's $t$ tests for the individual pairs of simulations and a subsequent meta-analysis based on Fisher's method have been conducted. The latter shows that the differences between the LEM and homogeneous simulations are indeed statistically significant, with $p$ values of $2.5 \times$ $10^{-3}$ for the entrainment velocity and $3.4 \times 10^{-12}$ for the in-cloud dissipation rate, that is, rejecting the null hypothesis (no difference between the LEM and the homogeneous simulations). This systematic difference can be attributed to the inability of the homogeneous simulations to represent SGS inhomogeneities of liquid water and supersaturation, forcing an entire grid box to evaporate at once, resulting in an accelerated evaporative cooling and hence stronger turbulence and entrainment. This will be analyzed further in section $3 b$. Note that the increase in the entrainment velocity due to details in the representation of SGS processes is comparable to the doubling of $N_{\text {init }}$. This shows that physics and numerics might have comparable effects, making direct comparisons of results obtained by different models problematic.

The strongest effect of the LEM is visible in $N_{c} / N_{\text {init }}$ (Fig. 2d), where the fraction of cloud droplets decreases from about $93 \%$ in the homogeneous simulations to about $87 \%$ in the LEM simulations, as also shown for a weakly turbulent cloud in Hoffmann et al. (2019). Since this reduces the number of water vapor competitors, $r_{\mathrm{eff}}$ increases slightly, most noticeably for $N_{\text {init }}=100 \mathrm{~cm}^{-3}$ (Fig. 2b). These distinct changes are characteristics of inhomogeneous mixing (e.g., Baker et al. 1980), and will be further analyzed in the next subsection. This, however, is in contrast to the simulations of Hill et al. (2009), who showed that inhomogeneous mixing has a negligible effect on the droplet number concentration in stratocumulus (see below).

\section{a. Microphysics}

Figure 3 shows the probability density function (PDF) of the droplet radius (Fig. 3a) and mean droplet-ageaveraged standard deviation of the absolute perturbation supersaturation $\sigma_{\delta^{\prime}}$ (Fig. 3b). The droplet-age average is determined for each superdroplet as

$$
\left\langle\Phi_{n}\right\rangle_{\mathrm{age}}=\frac{\int_{t_{\mathrm{act}}}^{t_{\mathrm{obs}}} \Phi\left[X_{i, n}(t)\right] d t}{t_{\mathrm{age}}},
$$

where $\Phi\left[X_{i, n}(t)\right]$ is an arbitrary quantity at the location of superdroplet $n$ at time $t$, and the droplet age is

$$
t_{\mathrm{age}}=\int_{t_{\mathrm{act}}}^{t_{\mathrm{obs}}} d t
$$

where $t_{\text {act }}$ is the time at which the superdroplet exceeds a radius of $1 \mu \mathrm{m}$, and $t_{\mathrm{obs}}$ is the time of the evaluation of 

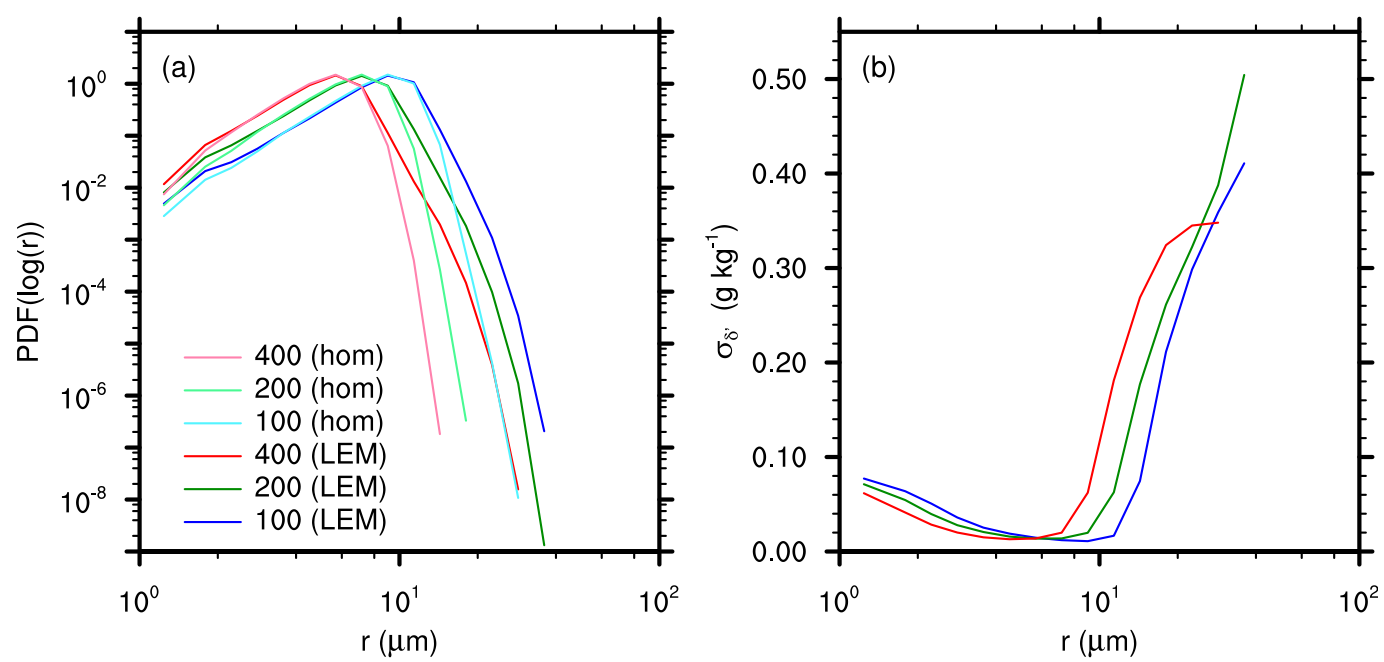

FIG. 3. Representation of (a) radius probability density function and (b) droplet-age-averaged standard deviation of absolute perturbation supersaturation as a function of the droplet radius. Data are averaged over the last $2 \mathrm{~h}$ of the simulation. See (a) for color code.

the desired quantity with the requirement that the superdroplet be larger than $1 \mu \mathrm{m}$ in radius. Note that $\sigma_{\delta^{\prime}}$ is calculated analogously to (7) without normalization by the saturation supersaturation. All quantities are calculated for the last two hours of the simulation over the entire model domain; that is, they include data from all droplets at various stages of their development.

All PDFs of the droplet radius exhibit one distinct mode that shifts to larger radii when $N_{\text {init }}$ is decreased. In contrast to the homogeneous simulations, all LEM simulations show a much broader spectrum, including droplets larger than $20 \mu \mathrm{m}$ in radius, which are usually able to trigger collision and coalescence (neglected for this study). The comparison of LEM and homogeneous simulations shows clearly that this broadening is created because of the explicit consideration of SGS supersaturation fluctuations by the LEM. This is in agreement with the distribution of $\sigma_{\delta^{\prime}}$, showing peak values for the largest radii, indicating that these droplets have spent some time in regions of inhomogeneous mixing at cloud top (see also Fig. 1d). Additionally, the share of the smallest droplets is also slightly increased in the LEM simulations. The distribution of $\sigma_{\delta^{\prime}}$ reinforces that also these smallest droplets have spent some time in regions of inhomogeneous mixing. Because of the explicit consideration of SGS supersaturation fluctuations by the LEM, SGS cloudy filaments in the subsaturated vicinity of the gridscale cloud are represented, preventing immediate evaporation when droplets are moved from the cloud. For the majority of droplets, however, SGS supersaturation fluctuations by the LEM are negligible since $\sigma_{\delta^{\prime}}$ is approximately zero.
Now, the same droplets are analyzed. However, the analysis is based on their age $t_{\text {age }}$, and not their radius as done above. Figure 4 displays the PDF of $t_{\text {age }}$ (Fig. 4a), mean droplet radius after $t_{\text {age }}$ (Fig. 4b), mean dropletage-averaged droplet height (Fig. 4c), and mean dropletage-averaged $\sigma_{\delta^{\prime}}$ (Fig. 4d). On the abscissa, $t_{\text {age }}$ is normalized by the cloud-layer eddy turnover time $\tau_{c}$, estimating the typical maximum droplet age based on the stratocumulus large-eddy circulation (e.g., Feingold et al. 1996; Kogan 2006). Time $\tau_{c}$ is defined as

$$
\tau_{c}=\frac{z_{t}-z_{b}}{\sigma_{w}},
$$

where $\sigma_{w}$ is the cloud-layer-averaged standard deviation of the vertical velocity, and $z_{b}$ and $z_{t}$ are the domainaveraged heights of cloud base and cloud top, respectively. The values of $z_{b}$ and $z_{t}$ are determined from lowest or highest cloudy grid cell in each column $\left(q_{c}>0.01 \mathrm{~g} \mathrm{~kg}^{-1}\right)$, respectively, and are located at on average at about $625 \pm 10 \mathrm{~m}$ and $830 \pm 10 \mathrm{~m}$. This difference can be attributed mainly to changes in cloud depth as a result of the evaporation- and sedimentationentrainment feedbacks in the simulated cases [see Figs. $1 \mathrm{a}(2)$ and $1 \mathrm{~b}(2)]$. Thus, $\tau_{c}$ varies between 380 (for $N_{\text {init }}=400 \mathrm{~cm}^{-3}$ ) and $420 \mathrm{~s}$ (for $N_{\text {init }}=100 \mathrm{~cm}^{-3}$ ). Note that the wiggles in the PDFs of $t_{\text {age }}$ at $t_{\text {age }} / \tau_{c}<10^{-2}$ are a result of undersampling (violation of the Nyquist sampling criterion) caused by the model's finite time step.

In Fig. 4a, all PDFs of $t_{\text {age }}$ exhibit an exponential shape, as also found by de Lozar and Muessle (2016) for the residence time of droplets at the top of stratocumulus simulated by DNS. The mode of all distributions 

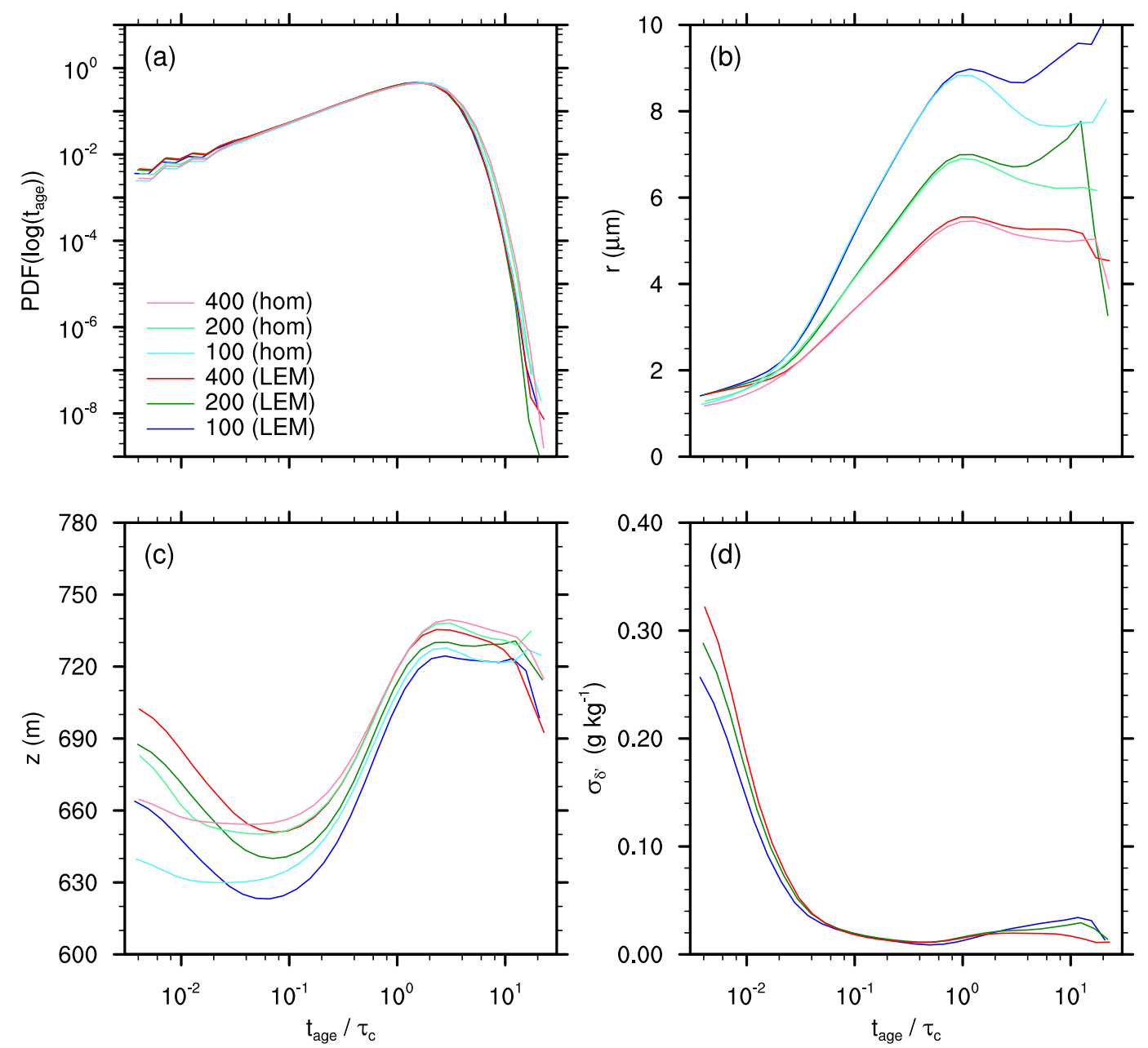

FIG. 4. Representation of (a) droplet-age probability density function, (b) radius at observation, (c) droplet-ageaveraged droplet height, and (d) droplet-age-averaged standard deviation of absolute perturbation supersaturation as a function of the normalized droplet age. Data are averaged over the last $2 \mathrm{~h}$ of the simulation. See (a) for color code.

is located at slightly longer droplet ages than $\tau_{c}$, which also marks, approximately, the timing of the maximum droplet radius (Fig. 4b) and the maximum droplet height (Fig. 4c). This temporal coincidence is not coincidental. Using an idealized picture of the dynamics of stratocumulus-topped boundary layers, where large eddies extend from the surface to the cloud top, $\tau_{c}$ is the time a droplet requires to be transported from the cloud base to the cloud top, where the maximum adiabatic radius also occurs. The steep decline in the PDF for $t_{\text {age }}>2 \tau_{c}$ shows that large eddies transport the majority of droplets below the cloud layer, where they evaporate eventually. Note that the general offset in the mean droplet height depending on $N_{\text {init }}$ (Fig. 4c) is a result of changes in the cloud depth due to the evaporation- and sedimentationentrainment feedbacks as discussed above.

However, Fig. 4 is slightly more complicated because of droplets that do not follow the large-eddy circulation. For example, the droplet radius increases until $\tau_{c}$, but does not decrease monotonically afterward. Similarly, the mean droplet height decreases only moderately after reaching its maximum. This behavior can be attributed to droplets that escape the large eddies, allowing them to stay within the cloud layer for up to $20 \tau_{c}$. In fact, the small changes in radius and mean height after their maximum at about $\tau_{c}$ indicate that all droplets with $t_{\mathrm{age}}>2 \tau_{c}$ move randomly inside the cloud layer, averaging out any individual differences.

Inhomogeneous mixing results in a distinct increase of the radius of droplets with $t_{\text {age }}>\tau_{c}$, as easily deduced by comparing the LEM and homogeneous simulations in Fig. 4b, and supported by Fig. 4d. Note that this effect decreases for larger $N_{\text {init }}$, where stronger turbulence results in more homogeneous mixing [see Figs. 1c(2) and 1d(2)]. Counterintuitively, this positive effect of inhomogeneous 
mixing on droplet growth actually decreases the number of old droplets (Fig. 4a) since they sediment from the cloud more quickly, resulting in earlier evaporation.

Another example of droplets that do not follow the simple pattern of large-eddy dynamics can be seen in the evolution of the mean droplet height for small $t_{\text {age }}$, that is, droplets that just started to grow after entering the cloud. As shown in Fig. 4c, the mean droplet height decreases initially and reaches its minimum at about $0.1 \tau_{c}$ before increasing again in accordance with the simple dynamical pattern of large eddies. The initially higher mean droplet height must result from droplets that did not start their growth history at cloud base, but at higher levels, that is, at cloud top or within cloud holes. However, these droplets evaporate again quickly, as indicated by the decrease in the mean droplet height prior to $0.1 \tau_{c}$. In other words, the contribution of these extraordinary droplets to the mean droplet height decreases rapidly because of their short lifetime. This confirms that particles entrained above the cloud base do not encounter an environment that supports longlasting diffusional growth for the production of longlived droplets (e.g., Hoffmann et al. 2015). On the other hand, the inhomogeneous simulations exhibit slightly higher mean droplet heights and slightly larger radii prior to $0.1 \tau_{c}$. This indicates that the successful representation of inhomogeneous mixing improves the harsh, competitive environment encountered after entrainment above cloud base by spatially separating the newly entrained particles from the larger old droplets, thus mitigating the direct competition for water vapor. As a result, the PDF of droplets with an age of up to $0.1 \tau_{c}$ is slightly higher in the LEM simulations compared to the homogeneous simulations (Fig. 4a).

\section{b. Dynamics}

The impact of inhomogeneous mixing on the dynamics of entrainment is now analyzed, focusing on cloud holes that transport free-tropospheric air into the cloud layer. Horizontally adjacent grid boxes with a liquid water mixing ratio of less than $0.01 \mathrm{~g} \mathrm{~kg}^{-1}$ and an average negative vertical velocity are identified as cloud holes. Connections in the vertical are not considered. Note that other authors may define cloud holes more broadly as volumes of depleted liquid water (e.g., Gerber et al. 2005; Yamaguchi and Randall 2012). Our analysis, however, should be viewed as an investigation on the initial stage of cloud holes, being on average subsaturated and most likely prone to inhomogeneous mixing, which is the main focus of this study. Furthermore, the restriction to cloud holes of negative vertical velocities only alters the results close to cloud base where some cloud holes exhibit slightly positive vertical velocities. Cloud holes are analyzed from snapshots taken every five minutes during the last two hours of the simulation.

Figure 5 shows vertical profiles of the number of cloud holes (Fig. 5a), average cloud-hole area $A$ normalized by the area of the entire model domain $\left(\sim 11.3 \mathrm{~km}^{2}\right)$ (Fig. 5b), cloud-hole vertical velocity (Fig. 5c), cloudhole buoyancy (Fig. 5d), and cloud-hole supersaturation (Fig. 5e). Note that the following analysis of vertical profiles can be understood as a time series of the evolution of cloud holes originating at the cloud top. This interpretation is justified since the cloud holes show negative velocities throughout the cloud (except for some cloud holes at the cloud base that have been neglected here). For comparison with the environment surrounding cloud holes, the vertical velocity and buoyancy are sampled over saturated (i.e., liquid water containing) downdrafts, which are displayed as black lines in Figs. 5c and 5d. For clarity, these are only shown for the inhomogeneous simulation with $N_{\text {init }}=200 \mathrm{~cm}^{-3}$, but do not vary significantly across the simulations conducted.

The maximum number of cloud holes is registered just below the average cloud-top height $(820 \mathrm{~m})$, while a small number of cloud holes is also registered above the average cloud-top height $(820-860 \mathrm{~m})$, framing the entrainment interface layer (Fig. 5a). All these cloud holes above $820 \mathrm{~m}$ are relatively large (Fig. 5b) and exhibit almost no vertical motion (Fig. 5c), but have the strongest negative buoyancy (Fig. 5d). This suggests that radiatively and evaporatively cooled air is accumulated in valleys at the cloud top before it descends into the cloud layer in the form of cloud holes (e.g., Rothermel and Agee 1980; Yamaguchi and Randall 2012). These cloud holes might not penetrate much farther into the cloud layer, as deduced from the small downward velocity and the diminishing negative buoyancy just below the cloud top (Figs. 5c,d) (e.g., Gerber et al. 2005; Haman 2009).

Within the cloud layer, the downward motion of the newly engulfed cloud holes accelerates, and their average area reduces, because of their mixing and homogenization with the cloud, reducing the number of cloud holes with increasing distance to the cloud top as also seen in observations (e.g., Nicholls 1989). As a result of the mixing, evaporative cooling causes a second minimum in buoyancy at $\sim 780 \mathrm{~m}$, excluding additional radiative cooling as an explanation, because of its location inside the cloud. This behavior supports the so-called cloud interior mixing instability as hypothesized by Gerber et al. (2005) and further analyzed by Haman (2009) to explain the downward motion of cloud holes inside stratocumulus. However, this is not the only force 

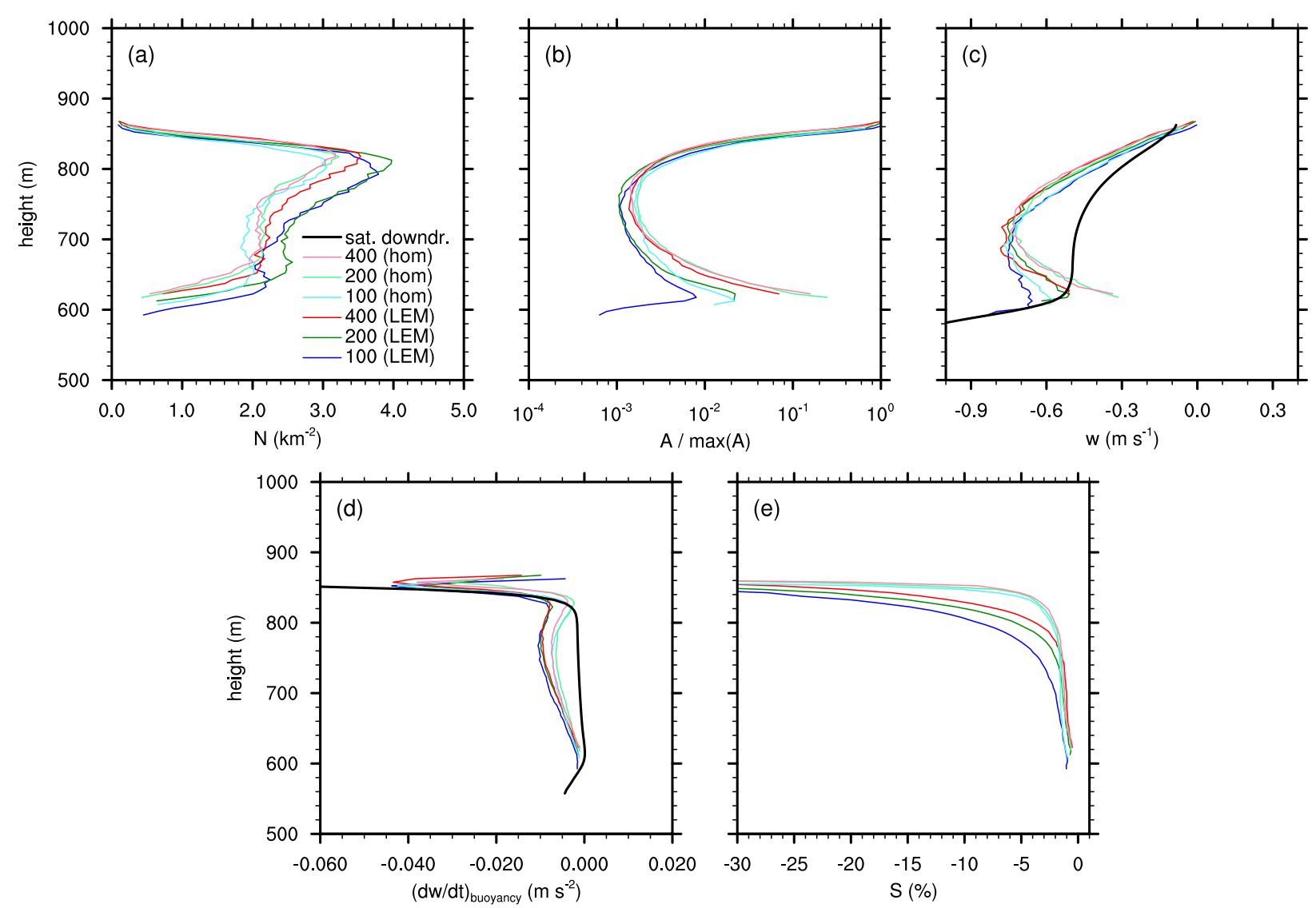

FIG. 5. Vertical profiles of (a) cloud-hole number, (b) average cloud-hole size, (c) cloud-hole vertical velocity, (d) cloud-hole buoyancy, and (e) cloud-hole supersaturation. Data are averaged over the last $2 \mathrm{~h}$ of the simulation. The black line shows selected quantities in saturated downdrafts of the inhomogeneous simulation with $N_{\text {init }}=200 \mathrm{~cm}^{-3}$. See (a) for color code.

responsible for the downward motion of cloud holes since they usually follow the subsiding branches of the stratocumulus large-eddy circulation (Yamaguchi and Randall 2012). In fact, this is also supported by our simulations, which show substantial downward motion in the downdrafts surrounding cloud holes (black line in Fig. 5c), indicating pressure gradient forces that move cloud holes deeper into the cloud layer. Accordingly, the mixing of the cloud hole with its environment and the resulting evaporative cooling within the cloud layer, that is, the cloud interior mixing instability, as well as the general downward motion determined by the stratocumulus large-eddy circulation act together to cause the downward motion of cloud holes.

The ongoing mixing and subsequent closing of cloud holes is also evident from the increase of the average cloud-hole area below $\sim 780 \mathrm{~m}$, a result of the closing of the smallest cloud holes, leaving only the largest (Fig. 5c). The closing of cloud holes during their descent is also evident in the cloud-hole supersaturation, which exhibits large negative values at the cloud top but becomes progressively more saturated toward cloud base (Fig. 5 e). The supersaturation is calculated as

$$
S=\bar{S}+\mu_{S^{\prime}},
$$

consisting of the LES supersaturation, $\bar{S}$, and the mean SGS supersaturation determined by the LEM, $\mu_{S^{\prime}}$, which is zero for the homogeneous simulations $\left(\mu_{S^{\prime}}=0\right)$. Thus, the cloud-hole supersaturation is a proxy for the overall force driving evaporation or condensation in a cloud hole, since both the LES and the LEM supersaturation are considered in the diffusional growth equation of a superdroplet, (3). Most evidently, the cloud holes in the LEM simulations do not saturate as fast as in the homogeneous simulations. There are two reasons for this. First, the LEM resolves the SGS distribution of supersaturation and liquid water much better than the LES. As a result, evaporation is restricted to the appropriate fraction of the grid box and not to the entire grid box as in the homogeneous simulation. This leads to a decelerated, more realistic temporal evolution of the mixing process, as also shown by Krueger (1993) in 
stand-alone simulations of the LEM. Second, numerical diffusion of supersaturation is reduced in the LEM simulations, removing this spurious source for evaporation to a large extent and decelerating the mixing process. In fact, de Lozar and Mellado (2015) showed that numerical diffusion dominates evaporation in (standard) LES, a problem that is mitigated by the new L3 modeling approach. All in all, the boundary of cloud holes is preserved much better using L3, resulting in a higher number of cloud holes (Fig. 5a), a generally lower cloud-hole saturation (Fig. 5e), and a weaker second minimum of buoyancy at $\sim 780 \mathrm{~m}$ (Fig. 5d). The latter difference might also indicate that the cloud interior mixing instability, and the resulting acceleration of cloud holes within the cloud layer, is overestimated in (standard) LESs.

Moreover, the cloud-hole supersaturation exhibits a much stronger susceptibility to the cloud droplet concentration in the LEM simulations than in the homogeneous simulations in which the profiles are almost indistinguishable (Fig. 5d). In general, it is plausible to assume that the cloud-hole supersaturation depends on the cloud droplet concentration. An increase in the cloud droplet concentration results not only in an accelerated evaporation of the droplet ensemble allowing depletion of the cloud-hole subsaturation more quickly (i.e., a shorter $\tau_{\text {phase }}$ ), but it also results in a faster decay of cloud holes due to stronger turbulence (i.e., a shorter $\tau_{\text {mix }}$ ), which are both the basis of the aforementioned evaporation-entrainment feedback (Wang et al. 2003). Although it is generally possible to represent these effects in the homogeneous simulations, it is clearly more pronounced in the LEM simulations. The reason for this is, again, the explicit resolution of SGS processes in the LEM simulations that restrict evaporation to an appropriate fraction of the grid box based on the SGS distribution of supersaturation and liquid water, which decelerates the mixing process (Krueger 1993). In other words, the LEM simulations resolve SGS inhomogeneous mixing sufficiently, while all droplets inside a grid box evaporate homogeneously in the homogeneous simulation. This can be easily understood by contrasting the effects of homogeneous and inhomogeneous mixing on the phase relaxation time scale as defined in (2). The liquid water mixing ratio after mixing (subscript "mix") is related to the initial value (subscript "init") via

$$
q_{c, \text { mix }}=\alpha q_{c, \text { init }} \propto \alpha N_{\text {init }} r_{v, \text { init }}^{3},
$$

where $0 \leq \alpha \leq 1$ indicates the mixing fraction and $r_{v}$ is the mean volume radius. For simplicity, we assume a monodisperse droplet spectrum in which the arithmetic and volume mean are identical (i.e., $r_{\mathrm{m}}=r_{v} \equiv r$ ). Under extreme inhomogeneous mixing, the mean radius does not change $\left(r_{\text {mix }}=r_{\text {init }}\right)$, and, consequently, $N_{\text {mix }}=\alpha N_{\text {init }}$. Under homogeneous mixing, the droplet number concentration is maintained $\left(N_{\text {mix }}=N_{\text {init }}\right)$, and, consequently, $r_{\text {mix }}=\alpha^{1 / 3} r_{\text {init. }}$. Thus, the phase relaxation time scale after mixing yields

$$
\tau_{\text {phase,mix }}=\tau_{\text {phase,init }} \alpha^{p},
$$

with $p=-1$ for extreme inhomogeneous mixing and $p=-1 / 3$ for homogeneous mixing. Thus, $\tau_{\text {phase,mix }}$ increases faster during inhomogeneous mixing than during homogeneous mixing, delaying the time necessary to saturate a volume (Fig. 5e). Note that $p=-1 / 3$ is the upper limit for homogeneous mixing, and it is closer to the value for extreme inhomogeneous mixing when a nonmonodisperse, positively skewed spectrum is considered, for which the arithmetic mean radius decreases faster than the mean volume radius.

Figure 6 shows the cloud-hole size distribution (Fig. 6a), cloud-hole vertical velocity (Fig. 6b), cloudhole buoyancy (Fig. 6c), and cloud-hole supersaturation as a function of the square root of the cloud-hole area, $A^{1 / 2}$ (Fig. 6d). Since cloud-hole characteristics vary greatly with height (Fig. 5), analysis is restricted to a height interval from 690 to $760 \mathrm{~m}$, that is, the center third of the cloud layer, to provide sufficient sampling statistics, while maintaining a relatively small vertical extent of the analyzed slab.

The cloud-hole size distribution (Fig. 6a) shows that the number of cloud holes follows a power law. In fact, it follows the same relationship identified by Yamaguchi and Feingold (2013) derived for the same stratocumulus case $\left[n(A) \propto A^{-1.68}\right]$. They used, however, a significantly smaller grid spacing and a different cloud microphysics scheme, suggesting that cloud holes are created on larger scales not affected by these details. The powerlaw relation breaks for small cloud holes $\left(A^{1 / 2}<80 \mathrm{~m}\right)$ and large cloud holes $\left(A^{1 / 2}>400 \mathrm{~m}\right)$. As discussed by Yamaguchi and Feingold (2013), the scale break for larger cloud holes is related to insufficient statistics as a result of the limited domain size. Although Yamaguchi and Feingold (2013) argue that the power-law relation can be extended to the smallest scales, which is true if the mixing were inhomogeneous down to the smallest scales, it becomes homogeneous below a certain length scale of a couple of centimeters to decimeters depending on turbulence and microphysical composition as determined by (5) (Baker et al. 1980; Lehmann et al. 2009). In the homogeneous simulations, this length scale is artificially increased to the LES grid spacing, and accordingly the slope is much more gradual for the smallest cloud holes than in the LEM simulations, and closer to the 

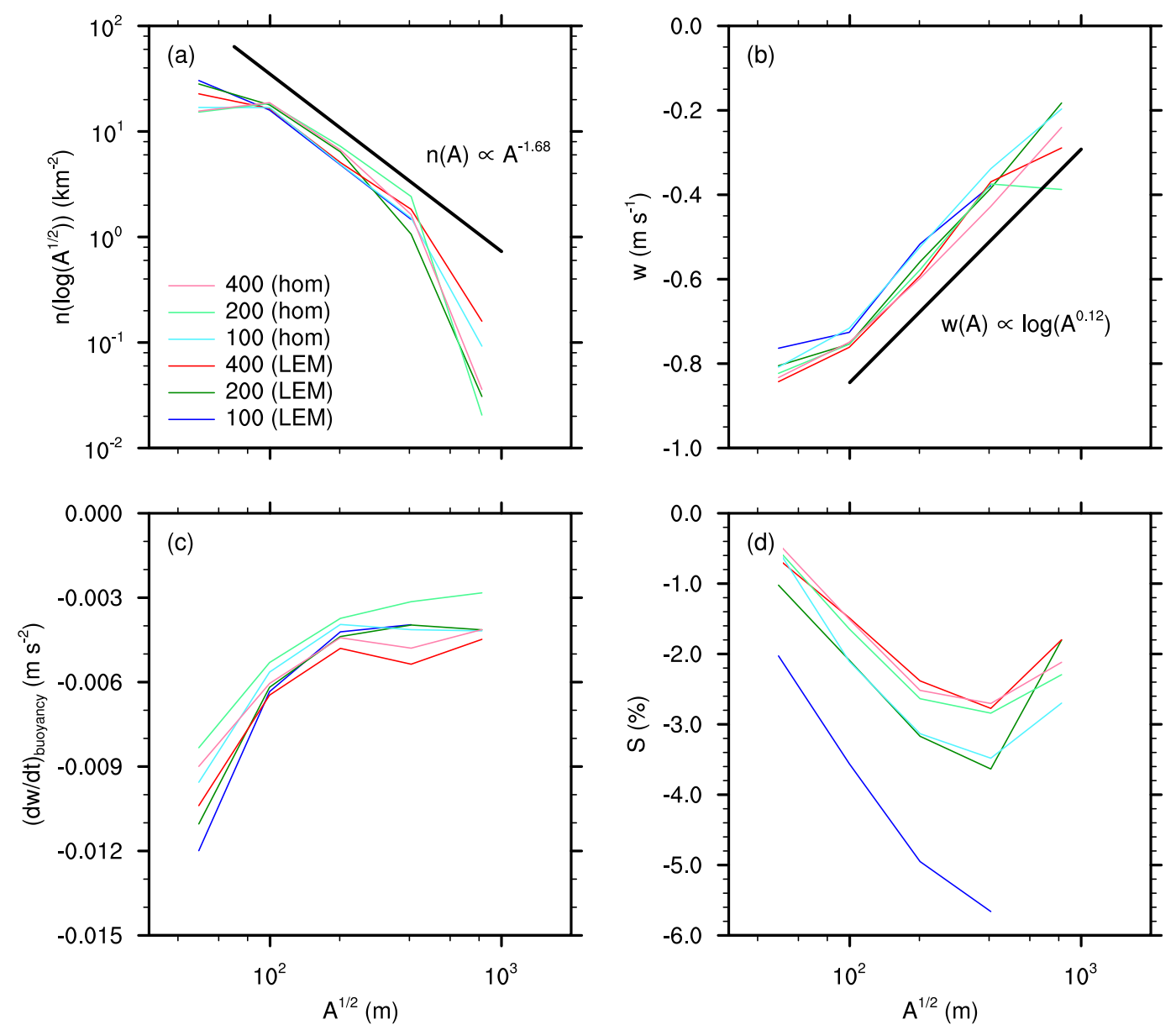

FIG. 6. Representation of (a) cloud-hole number, (b) cloud-hole vertical velocity, (c) cloud-hole buoyancy, and (d) cloud-hole supersaturation as a function of the cloud length $A^{1 / 2}$. Data are restricted to a height interval of 690$760 \mathrm{~m}$ and averaged over the last $2 \mathrm{~h}$ of the simulation. The thick black lines show fits to the analyzed data as a function of the cloud-hole area $A$. See (a) for color code.

slope for larger cloud holes, showing that SGS inhomogeneous mixing is better resolved in the LEM simulations. For these LEM simulations, the slope for the smallest cloud holes also depends on the number of cloud droplets, becoming more gradual in more dropletladen clouds. This is, again, a result of the evaporationentrainment feedback that accelerates the mixing of cloud holes and cloudy air and therefore increases the length scale at which mixing becomes homogeneous. Note that the changes in the distribution of the cloud holes among the different simulations are restricted to the smallest resolved cloud holes. Accordingly, changes in the total number of cloud holes as depicted in the profiles of Fig. 5a are a result of changes in the number of the smallest cloud holes while the number of larger cloud holes is largely the same in all cases.

The cloud-hole vertical velocity (Fig. 6b) and the cloud-hole buoyancy (Fig. 6c) show a distinct decrease for smaller cloud holes, where the vertical velocity again follows a simple relationship of $w(A) \propto \log \left(A^{0.12}\right)$. One can hypothesize that the acceleration of the downward vertical velocity in smaller cloud holes is a result of the increasing perimeter to area ratio for smaller cloud holes, causing a stronger relative impact of mixing and evaporative cooling and hence a larger negative buoyancy for the smallest cloud holes. Note that Yamaguchi and Randall (2012) show that the final entrainment of cloud holes into the boundary layer takes place when buoyancy matches, while our results show that the smallest cloud holes exhibit a buoyancy that is significantly different from their environment (see also Fig. 5d). This difference may be explained by the slightly divergent definitions of a cloud hole and hence their entrainment: we consider a cloud hole to be homogenized with the cloud once it contains liquid water, while Yamaguchi and Randall (2012) require more restrictively that moist conserved scalars match, which usually occurs after the cloud hole contains some liquid water. In other words, 
the entrainment process is not complete when we stop tracking cloud holes.

The distribution of the cloud-hole supersaturation (Fig. 6d), however, cannot be understood by the relatively increased evaporative cooling for the smallest cloud holes alone; the downward motion (and hence adiabatic heating rates) needs to be considered. The downward motion increases for smaller cloud holes; however, the smallest cloud holes are the least subsaturated. This indicates that the effect of adiabatic heating on the cloud-hole supersaturation dominates only for the largest cloud holes, and evaporative cooling for the smallest, resulting in a cloudhole supersaturation minimum $\left(A^{1 / 2} \approx 400 \mathrm{~m}\right)$, at which the dominance of adiabatic heating and evaporative cooling on the cloud-hole supersaturation switches.

Although mixing accelerates for higher droplet concentrations and hence evaporative cooling, the cloud-hole buoyancy shows an almost inverse pattern: LEM simulations with a low droplet number exhibit the most negative buoyancy while droplet-laden simulations display slightly higher (less negative) values. This does not contradict accelerated evaporation cooling in droplet-laden conditions, but rather shows that most of the evaporative cooling has already happened at greater heights, limiting further evaporative cooling below (Fig. 6d). This is also supported by the height of the second minimum of the cloud-hole buoyancy in Fig. 5d, which is located at higher levels for higher droplet concentrations. Overall, this indicates that mixing in droplet-laden clouds is faster, and, for the most part, takes place at higher levels in the cloud, that is, closer to the cloud top. On the other hand, this means that cloud holes are able to penetrate deeper into the cloud if the droplet number concentration is low.

Finally, the contribution of cloud-hole sizes to entrainment needs to be addressed. As argued by Nicholls (1989) and Gerber et al. (2005), the entrainment velocity is proportional to the product of the (absolute) cloudhole vertical velocity and the total area covered by cloud holes. Since this relationship also holds for a certain cloud-hole size, the power-law fits derived for Figs. 6a and $6 \mathrm{~b}$ indicate that the largest fraction of entrainment originates from the smallest cloud holes [using $|w(A)| \propto \log \left(A^{-0.12}\right)$ for the (absolute) cloud-hole vertical velocity and $A n(A) \propto A^{-0.68}$ for the total area of cloud holes of a certain size]. This confirms the results of Yamaguchi and Feingold (2013), who showed by tracking Lagrangian parcels that the smallest cloud holes contribute most to entrainment.

\section{Discussion and conclusions}

In this study, the new L3 modeling approach for the explicit representation of turbulent mixing on the subgrid scale of LES with particle-based cloud microphysics is applied to investigate the entrainment and mixing process in stratocumulus and its effects on cloud microphysics. This new approach, which has been described by Hoffmann et al. (2019), applies the linear eddy model (LEM) by Kerstein (1988), an explicit, computationally inexpensive, one-dimensional representation of SGS turbulent compression and folding, as well as molecular diffusion, to replace the instantaneous, homogeneous mixing calculated in typical LES SGS models. The purpose of this study has been threefold: first, to understand how the application of L3 alters the representation of the physics of stratocumulus compared to the standard LES modeling approach; second, to increase the process-level understanding of entrainment and mixing, focusing on the now sufficiently resolved inhomogeneous mixing, which is the prevalent mixing type in stratocumulus (e.g., Pawlowska et al. 2000; Gerber et al. 2005) but not well represented in typical LES models since it requires a computationally infeasible grid spacing of less than a couple of centimeters to decimeters (Lehmann et al. 2009); and third, to investigate the effect of drop concentration on the entrainment and mixing processes.

Consistent with the theory of Baker and Latham (1979) on inhomogeneous mixing, the simulations presented show that the number of cloud droplets is reduced during mixing and that the remaining droplets grow to larger sizes because of the reduced number of water vapor competitors. This is in stark contrast to the results of Hill et al. (2009), who showed negligible changes in the number of cloud droplets and other variables even though extreme inhomogeneous mixing was enforced on the subgrid scale by scaling the microphysical variables accordingly. The reason for this disagreement is likely numerical diffusion that spuriously humidifies the entrained air in Hill et al.'s (2009) standard LES. Accordingly, the mixed air is almost saturated and the mixing process, although it is microphysically extremely inhomogeneous, does not result in a significant reduction of the droplet number concentration, and its effects cannot be distinguished from homogeneous mixing (Pinsky et al. 2016).

Furthermore, our results show that inhomogeneous mixing, interestingly, does not increase cloud droplet age, as might have been assumed since inhomogeneous mixing tends to prevent the evaporation of droplets at cloud edge. Instead, inhomogeneous mixing reduces the number of the oldest droplets since increased diffusional growth results in faster sedimentation, removing those droplets from the cloud more quickly.

The dynamics of the entrainment and mixing process have been investigated by focusing on the development 
of cloud holes. These holes, which transport freetropospheric air into the cloud layer, can be regarded as the initial step of the entrainment and mixing process (Nicholls 1989; Gerber et al. 2005; Yamaguchi and Randall 2012; Yamaguchi and Feingold 2013). It has been shown that cloud holes are much better represented using L3, as documented by their larger number and their generally higher subsaturation compared to simulations without the LEM as an SGS model. L3 is able to represent and maintain the distribution of liquid water and supersaturation on the subgrid scale, preventing spurious evaporation, but it is also able to reduce numerical diffusion of supersaturation, avoiding the spurious erosion of strong gradients between cloud holes and the cloud and hence preserving the structure of cloud holes. Overall, the improved representation of the entrainment process in L3 reduces the entrainment velocity by about $5 \%$ compared to simulations without the LEM as an SGS model. Accordingly, L3 mitigates, at least partially, problems associated with the spurious overestimation of entrainment in LES of stratocumulus with standard SGS models (e.g., Stevens et al. 2005; de Lozar and Mellado 2015; Mellado et al. 2018).

Furthermore, the study gives insights into the temporal development of cloud holes. Just above the cloud top, in the so-called entrainment interface layer, cloud holes exhibit their strongest negative buoyancy and lowest (most negative) vertical velocities, indicating that certain areas of the cloud top are filled with negatively buoyant air until the cloud top is sufficiently destabilized, enabling cloud holes to descend into the cloud layer (Rothermel and Agee 1980; Yamaguchi and Randall 2012). As shown by Yamaguchi and Randall (2012), cloud holes follow the downward branches of the stratocumulus large-eddy circulation once they have entered the cloud layer. Our analysis indicates that the ongoing mixing of cloud holes with their environment and the subsequent evaporative cooling contribute to the downward motion, as also hypothesized by Gerber et al. (2005) and further analyzed by Haman (2009) in terms of the cloud interior mixing instability. It is shown that this process is accelerated for clouds with higher droplet concentrations, in which the faster evaporation process increases turbulence, which further amplifies the mixing of the cloud hole with its environment, as explained in the evaporation-entrainment feedback introduced by Wang et al. (2003).

Note, in contrast to the cloud top, it is rather unlikely that the sedimentation-entrainment feedback (Ackerman et al. 2004; Bretherton et al. 2007) is responsible for this accelerated closing of cloud holes: To understand this, one can compare the velocity of cloud droplets to that of the surrounding fluid, and accordingly the relative time for interaction. At the cloud top, the droplet terminal velocity exceeds the motion of the cloud interface $\left(\sim 1 \mathrm{~cm} \mathrm{~s}^{-1}\right)$ once droplets are larger than $10 \mu \mathrm{m}$ in radius, resulting in a significant removal of liquid water from the cloud interface. Cloud holes, however, might exhibit a downward velocity of up to $0.3 \mathrm{~m} \mathrm{~s}^{-1}$ faster than the surrounding fluid, which is faster than the terminal velocity of the largest simulated droplets that are generally smaller than $40 \mu \mathrm{m}$, and accordingly fall slower than about $0.2 \mathrm{~m} \mathrm{~s}^{-1}$, reducing the potential for the sedimentation-entrainment feedback.

Finally, it is worthwhile noting that the evaporationentrainment feedback is more pronounced in the L3 simulations. The reason for this is the largely resolved inhomogeneous mixing, which results in a stronger increase in the phase relaxation time scale during inhomogeneous mixing compared to homogeneous mixing. This indicates that a significantly higher model resolution is necessary to represent this process in (standard) LES, as previously argued for the sedimentationentrainment feedback analyzed in DNS simulations by de Lozar and Mellado (2017).

Note that the horizontal LES grid spacing of $35 \mathrm{~m}$ used in this study is not able to explicitly resolve all cloud holes, which are most abundant at a size of about $5 \mathrm{~m}$, according to observations by Gerber et al. (2005). Nonetheless, the simulations presented here capture the main dynamics responsible for the production of cloud holes successfully, as indicated by the same power-law relationship for the cloud-hole size distribution as obtained in the higher-resolution modeling by Yamaguchi and Feingold (2013). Moreover, the explicit representation of the SGS distribution of supersaturation and liquid water by the LEM is able to partially compensate for this lack of resolution in the LES. However, the LEM does not include an SGS representation of buoyancy fluctuations and pressure gradients, which need to be included potentially, unless the LES resolution surpasses the Ozmidov scale, below which these processes can be neglected. Accordingly, further model development will be necessary to further improve the representation of the LES subgrid scale by the LEM. Furthermore, we would like to draw attention to the fact that we only predict $\delta_{n}^{\prime}$ in the SGS model, potentially underestimating supersaturation fluctuations resulting from the supersaturation's nonlinear dependence on temperature, as outlined in Hoffmann et al. (2019).

Since dynamical and microphysical effects are usually tightly connected, future studies to untangle the microand macroscale effects of the L3 approach layer more clearly are necessary. These could be based on the piggybacking approach (Grabowski 2014), or use a simple kinematic framework (e.g., Shipway and Hill 2012), 
which lacks the interaction with dynamics entirely. The latter approach would also allow for comparison of L3 directly with other SGS models for supersaturation fluctuations [e.g., the model by Grabowski and Abade (2017)]. Furthermore, future studies should also include the entire suite of relevant warm-cloud microphysics, that is, explicit droplet activation as well as collision and coalescence, which have been neglected in this study for simplicity and clarity. For example, the nonlinear response of droplet activation to supersaturation fluctuations (Abade et al. 2018) might have additional impacts on the number of activated droplets, in addition to the effects of inhomogeneous mixing investigated here.

All in all, this study shows that the new L3 approach enables, in a single framework, simulation of large domains and their large-scale physics appropriately, while also representing the small-scale physics of entrainment and mixing adequately. Therefore, L3 can be viewed as a first step to bridge the gap between LES and DNS.

Acknowledgments. This research was performed while F.H. held a Visiting Fellowship of the Cooperative Institute for Research in Environmental Sciences (CIRES) at the University of Colorado Boulder and the NOAA Earth System Research Laboratory. Marat Khairoutdinov graciously provided the SAM model. We thank two anonymous reviewers and Steve Krueger for their comments on the manuscript.

\section{APPENDIX}

\section{Treatment of Sedimentation}

Strictly speaking, the L3 modeling approach does not allow sedimentation since the superdroplets are not only used to represent cloud droplets but also to depict a volume of air surrounding these particles. In principle, it would be possible to allow droplets to sediment within the one-dimensional LEM array, as is done in standalone applications of the LEM (Su et al. 1998; Tölle and Krueger 2014), and to change the volume of air surrounding a superdroplet accordingly. This, however, is not possible here since sedimentation might result in the accumulation of superdroplets in one volume of air, while another volume of air might be devoid of any superdroplets. Of course, it would be possible to circumvent this problem by creating additional superdroplets for the tracking of the empty volumes of air. But this would increase computing time and memory demand, and potentially lead to load imbalance issues. To avoid this, only those superdroplets that sediment negligibly compared to the turbulent motion of the surrounding fluid are considered in the LEM of L3, while faster sedimenting superdroplets are excluded from the consideration in the LEM; that is, they are treated as in the homogeneous modeling approach with $\delta_{n}^{\prime}=0$.

To determine if droplet sedimentation can be neglected in comparison to the turbulent motion, the so-called velocity ratio is calculated for each superdroplet as

$$
S_{v}=\frac{w_{s}}{w_{\eta}}
$$

where $w_{s}$ is the droplet terminal velocity and $w_{\eta}$ is the physical Kolmogorov velocity scale (e.g., Vaillancourt et al. 2002). If $S_{v} \ll 1$, the turbulent motion exceeds the sedimentation velocity significantly, and sedimentation can be neglected. The physical Kolmogorov velocity scale is defined as

$$
w_{\eta}=(\nu \varepsilon)^{1 / 4},
$$

where $\nu$ is the kinematic viscosity, and $\varepsilon$ the kinetic energy dissipation rate. Since the LEM grid cell is usually larger than the physical Kolmogorov scale, $\nu$ needs to be scaled to the LEM Kolmogorov scale,

$$
\eta_{*}=6 \Delta z^{\prime}
$$

where $\Delta z^{\prime}$ is the length of an LEM grid cell, calculated as

$$
\Delta z^{\prime}=\frac{L}{N},
$$

where $L$ is the LES model's smallest gridscale eddy size, corresponding to the vertical grid spacing $\Delta z$ in the applied LES model, and $N$ is the number of superdroplets within an LES grid box, which determines the LEM's resolution.

As explained in Krueger et al. (1997), $\nu$ needs to be replaced by the LEM diffusion coefficient if the physical Kolmogorov length scale is not resolved:

$$
\begin{aligned}
D_{\mathrm{LEM}} & =D_{\mathrm{LES}}\left(\frac{\eta_{*}}{L}\right)^{4 / 3} \approx 0.1 L^{4 / 3} \varepsilon^{1 / 3}\left(\frac{\eta_{*}}{L}\right)^{4 / 3} \\
& =0.1 \varepsilon^{1 / 3} \eta_{*}^{4 / 3},
\end{aligned}
$$

where $D_{\mathrm{LES}}$ is the LES diffusion coefficient determined by the LES SGS model, which can be approximated as $D_{\text {LES }} \approx 0.1 L^{4 / 3} \varepsilon^{1 / 3}$ (Krueger 1993). Accordingly, the LEM Kolmogorov velocity scale yields

$$
w_{\eta_{*}} \approx 0.56 \varepsilon^{1 / 3} \eta_{*}^{1 / 3} .
$$

Note that the approximation of $D_{\text {LES }}$ is for illustrative purposes only; $D_{\text {LES }}$ is directly determined by the LES SGS model in the simulations presented above. 


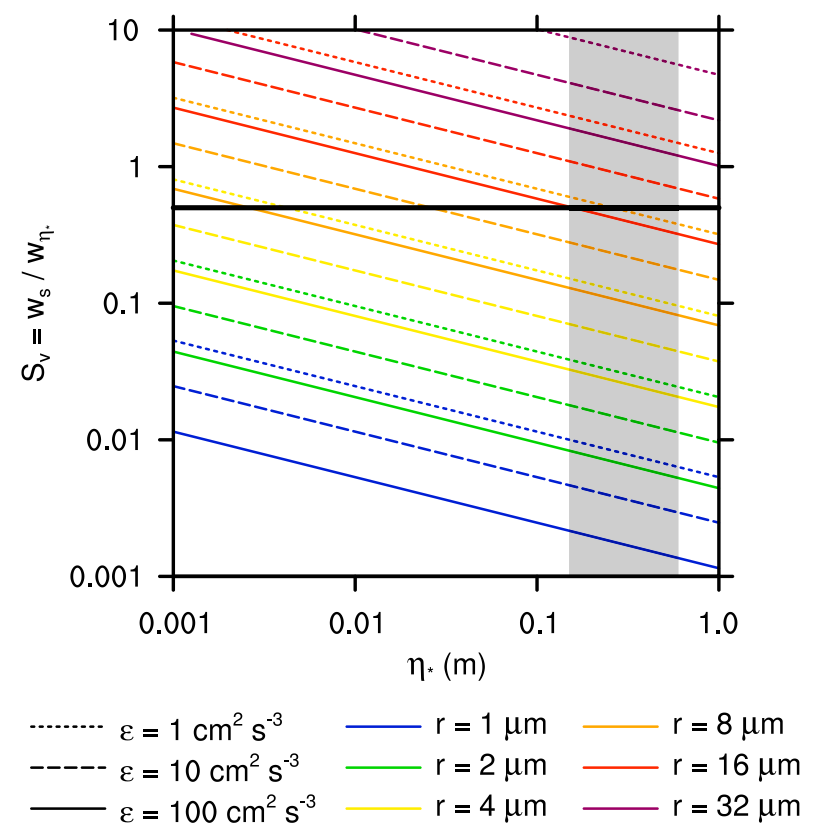

FIG. A1. Velocity ratio $S_{v}$ as a function of the model Kolmogorovscale $\eta_{*}$ for different kinetic energy dissipation rates (line pattern) and droplet radii (line color). The gray-shaded area indicates typical values for $\eta_{*}$, and the thick horizontal line indicates the critical value for $S_{v}=0.5$ used in this study.

By using (A6) and Beard (1976) for $w_{s}$, the velocity ratio $S_{v}$ is plotted as a function of $\eta_{*}$ in Fig. A1. The gray-shaded area indicates typical values for $\eta_{*}(30 \mathrm{~cm}$ for this study). The value $S_{v}=0.5$ (thick horizontal line) is used in all simulations presented above to decide if sedimentation is significant. This value is sufficiently small to neglect sedimentation safely (e.g., Shaw et al. 1999), and has been chosen to restrict the underestimation of mixing in the LEM for the largest droplets to $33 \%$ as outlined in the next paragraph. As a result of this choice, droplets with radii below $8 \mu \mathrm{m}$ are usually treated with the L3 modeling approach (depending on $\varepsilon$ ), while larger droplets experience the homogeneous LES supersaturation. The latter is a valid assumption for rapidly sedimenting droplets since they are exposed to quickly varying supersaturations during their fall, resulting in the experience of an effectively homogeneous supersaturation.

All in all, neglecting sedimentation potentially underestimates the mixing of sedimenting droplets in the LEM, which can be estimated as follows. Here, the velocity ratio $S_{v}$ will be interpreted as the ratio of the time scales for the mixing on the model Kolmogorov-scale $\tau_{\eta_{*}}$ to the sedimentation time scale $\tau_{s}$ :

$$
S_{v}=\frac{\tau_{\eta_{*}}}{\tau_{s}} .
$$

To estimate the total mixing time scale on the model Kolmogorov scale including the effect of sedimentation, both $\tau_{\eta_{*}}$ and $\tau_{s}$ need to be added as (Tölle and Krueger 2014)

$$
\begin{aligned}
\tau_{\eta_{*}, \text { tot }} & =\left(\tau_{\eta_{*}}^{-1}+\tau_{s}^{-1}\right)^{-1} \\
& =\left(\tau_{\eta_{*}}^{-1}+S_{v} \tau_{\eta_{*}}^{-1}\right)^{-1} \\
& =\tau_{\eta_{*}}\left(1+S_{v}\right)^{-1}
\end{aligned}
$$

Since a threshold of $S_{v}=0.5$ is used in all simulation, this expression yields a maximum underestimation of mixing by $33 \%$ for the largest droplets in the LEM and commensurately less for smaller droplets.

\section{REFERENCES}

Abade, G. C., W. W. Grabowski, and H. Pawlowska, 2018: Broadening of cloud droplet spectra through eddy hopping: Turbulent entraining parcel simulations. J. Atmos. Sci., 75, 3365-3379, https://doi.org/10.1175/JAS-D-18-0078.1.

Ackerman, A. S., M. P. Kirkpatrick, D. E. Stevens, and O. B. Toon, 2004: The impact of humidity above stratiform clouds on indirect aerosol climate forcing. Nature, 432, 1014-1017, https:// doi.org/10.1038/nature03174.

Andrejczuk, M., J. M. Reisner, B. Henson, M. K. Dubey, and C. A. Jeffery, 2008: The potential impacts of pollution on a nondrizzling stratus deck: Does aerosol number matter more than type? J. Geophys. Res., 113, D19204, https://doi.org/ 10.1029/2007JD009445.

Arabas, S., A. Jaruga, H. Pawlowska, and W. Grabowski, 2015: libcloudph ++1.0 : A single-moment bulk, double-moment bulk, and particle-based warm-rain microphysics library in $\mathrm{C}++$. Geosci. Model Dev., 8, 1677-1707, https://doi.org/10.5194/ gmd-8-1677-2015.

Baker, M., and J. Latham, 1979: The evolution of droplet spectra and the rate of production of embryonic raindrops in small cumulus clouds. J. Atmos. Sci., 36, 1612-1615, https://doi.org/ 10.1175/1520-0469(1979)036<1612:TEODSA > 2.0.CO;2.

, R. Corbin, and J. Latham, 1980: The influence of entrainment on the evolution of cloud droplet spectra: I. A model of inhomogeneous mixing. Quart. J. Roy. Meteor. Soc., 106, 581598, https://doi.org/10.1002/qj.49710644914.

Beard, K. V., 1976: Terminal velocity and shape of cloud and precipitation drops aloft. J. Atmos. Sci., 33, 851-864, https://doi.org/ 10.1175/1520-0469(1976)033<0851:TVASOC >2.0.CO;2.

Bretherton, C., P. N. Blossey, and J. Uchida, 2007: Cloud droplet sedimentation, entrainment efficiency, and subtropical stratocumulus albedo. Geophys. Res. Lett., 34, L03813, https:// doi.org/10.1029/2006GL027648.

Deardorff, J. W., 1980: Stratocumulus-capped mixed layers derived from a three-dimensional model. Bound.-Layer Meteor., 18, 495-527, https://doi.org/10.1007/BF00119502.

de Lozar, A., and J. P. Mellado, 2015: Mixing driven by radiative and evaporative cooling at the stratocumulus top. J. Atmos. Sci., 72, 4681-4700, https://doi.org/10.1175/JAS-D-15-0087.1.

— tocumulus top. Atmos. Chem. Phys., 16, 6563-6576, https:// doi.org/10.5194/acp-16-6563-2016. 
— , and J. P. Mellado, 2017: Reduction of the entrainment velocity by cloud droplet sedimentation in stratocumulus. J. Atmos. Sci., 74, 751-765, https://doi.org/10.1175/JAS-D-16-0196.1.

Feingold, G., and H. Siebert, 2009: Cloud-aerosol interactions from the micro to the cloud scale. Clouds in the Perturbed Climate System: Their Relationship to Energy Balance, Atmospheric Dynamics, and Precipitation, J. Heintzenberg and R. Charlson, Eds., Strüngmann Forum Reports, Vol. 2, MIT Press, 319-338.

_, W. Cotton, B. Stevens, and A. Frisch, 1996: The relationship between drop in-cloud residence time and drizzle production in numerically simulated stratocumulus clouds. J. Atmos. Sci., 53, 1108-1122, https://doi.org/10.1175/1520-0469(1996)053<1108: TRBDIC $>2.0 . \mathrm{CO} ; 2$.

Gerber, H., G. Frick, S. Malinowski, J. Brenguier, and F. Burnet, 2005: Holes and entrainment in stratocumulus. J. Atmos. Sci., 62, 443-459, https://doi.org/10.1175/JAS-3399.1.

Grabowski, W. W., 2014: Extracting microphysical impacts in large-eddy simulations of shallow convection. J. Atmos. Sci., 71, 4493-4499, https://doi.org/10.1175/JAS-D-14-0231.1.

— through eddy hopping: Turbulent adiabatic parcel simulations. J. Atmos. Sci., 74, 1485-1493, https://doi.org/10.1175/ JAS-D-17-0043.1.

_- P. Dziekan, and H. Pawlowska, 2018: Lagrangian condensation microphysics with Twomey CCN activation. Geosci. Model Dev., 11, 103-120, https://doi.org/10.5194/gmd-11-103-2018.

Haman, K. E., 2009: Simple approach to dynamics of entrainment interface layers and cloud holes in stratocumulus clouds. Quart. J. Roy. Meteor. Soc., 135, 93-100, https:// doi.org/10.1002/qj.363.

Hill, A. A., G. Feingold, and H. Jiang, 2009: The influence of entrainment and mixing assumption on aerosol-cloud interactions in marine stratocumulus. J. Atmos. Sci., 66, 1450-1464, https://doi.org/10.1175/2008JAS2909.1.

Hoffmann, F., 2017: On the limits of Köhler activation theory: How do collision and coalescence affect the activation of aerosols? Atmos. Chem. Phys., 17, 8343-8356, https://doi.org/10.5194/ acp-17-8343-2017.

- S. Raasch, and Y. Noh, 2015: Entrainment of aerosols and their activation in a shallow cumulus cloud studied with a coupled LCM-LES approach. Atmos. Res., 156, 43-57, https:// doi.org/10.1016/j.atmosres.2014.12.008.

_ , Y. Noh, and S. Raasch, 2017: The route to raindrop formation in a shallow cumulus cloud simulated by a Lagrangian cloud model. J. Atmos. Sci., 74, 2125-2142, https://doi.org/10.1175/ JAS-D-16-0220.1.

_- T. Yamaguchi, and G. Feingold, 2019: Inhomogeneous mixing in Lagrangian cloud models: Effects on the production of precipitation embryos. J. Atmos. Sci., 76, 113-133, https:// doi.org/10.1175/JAS-D-18-0087.1.

Jarecka, D., W. W. Grabowski, and H. Pawlowska, 2009: Modeling of subgrid-scale mixing in large-eddy simulation of shallow convection. J. Atmos. Sci., 66, 2125-2133, https://doi.org/ 10.1175/2009JAS2929.1.

Jeffery, C. A., 2007: Inhomogeneous cloud evaporation, invariance, and Damköhler number. J. Geophys. Res., 112, D24S21, https:// doi.org/10.1029/2007JD008789.

Kerstein, A. R., 1988: A linear-eddy model of turbulent scalar transport and mixing. Combust. Sci. Technol., 60, 391-421, https://doi.org/10.1080/00102208808923995.

Khairoutdinov, M. F., and D. A. Randall, 2003: Cloud resolving modeling of the ARM summer 1997 IOP: Model formulation, results, uncertainties, and sensitivities. J. Atmos. Sci., 60, 607-625, https://doi.org/10.1175/1520-0469(2003)060<0607:CRMOTA $>$ 2.0.CO;2.

Kogan, Y. L., 1991: The simulation of a convective cloud in a 3-D model with explicit microphysics. Part I: Model description and sensitivity experiments. J. Atmos. Sci., 48, 1160-1189, https://doi.org/10.1175/1520-0469(1991)048<1160:TSOACC $>$ 2.0.CO;2.

_ 2006 : Large-eddy simulation of air parcels in stratocumulus clouds: Time scales and spatial variability. J. Atmos. Sci., 63, 952-967, https://doi.org/10.1175/JAS3665.1.

Krueger, S. K., 1993: Linear eddy modeling of entrainment and mixing in stratus clouds. J. Atmos. Sci., 50, 3078-3090, https:// doi.org/10.1175/1520-0469(1993)050<3078:LEMOEA > 2.0.CO;2.

— C.-W. Su, and P. A. McMurtry, 1997: Modeling entrainment and finescale mixing in cumulus clouds. J. Atmos. Sci., 54, 2697-2712, https://doi.org/10.1175/1520-0469(1997)054<2697: MEAFMI $>2.0 . \mathrm{CO} ; 2$.

Lehmann, K., H. Siebert, and R. A. Shaw, 2009: Homogeneous and inhomogeneous mixing in cumulus clouds: Dependence on local turbulence structure. J. Atmos. Sci., 66, 3641-3659, https:// doi.org/10.1175/2009JAS3012.1.

Lilly, D. K., 1968: Models of cloud-topped mixed layers under a strong inversion. Quart. J. Roy. Meteor. Soc., 94, 292-309, https://doi.org/10.1002/qj.49709440106.

Magaritz-Ronen, L., M. Pinsky, and A. Khain, 2014: Effects of turbulent mixing on the structure and macroscopic properties of stratocumulus clouds demonstrated by a Lagrangian trajectory model. J. Atmos. Sci., 71, 1843-1862, https://doi.org/ 10.1175/JAS-D-12-0339.1.

Mellado, J. P., 2017: Cloud-top entrainment in stratocumulus clouds. Annu. Rev. Fluid Mech., 49, 145-169, https://doi.org/ 10.1146/annurev-fluid-010816-060231.

— C. Bretherton, B. Stevens, and M. Wyant, 2018: DNS and LES for simulating stratocumulus: Better together. $J$. $A d v$. Model. Earth Syst., 10, 1421-1438, https://doi.org/10.1029/ 2018MS001312.

Mordy, W., 1959: Computations of the growth by condensation of a population of cloud droplets. Tellus, 11, 16-44, https://doi.org/ 10.3402/tellusa.v11i1.9283.

Nicholls, S., 1989: The structure of radiatively driven convection in stratocumulus. Quart. J. Roy. Meteor. Soc., 115, 487-511, https://doi.org/10.1002/qj.49711548704.

Pawlowska, H., J. Brenguier, and F. Burnet, 2000: Microphysical properties of stratocumulus clouds. Atmos. Res., 55, 15-33, https://doi.org/10.1016/S0169-8095(00)00054-5.

Pedersen, J. G., S. P. Malinowski, and W. W. Grabowski, 2016: Resolution and domain-size sensitivity in implicit large-eddy simulation of the stratocumulus-topped boundary layer. J. Adv. Model. Earth Syst., 8, 885-903, https://doi.org/10.1002/2015MS000572.

Pinsky, M., A. Khain, and A. Korolev, 2016: Theoretical analysis of mixing in liquid clouds-Part 3: Inhomogeneous mixing. Atmos. Chem. Phys., 16, 9273-9297, https://doi.org/10.5194/ acp-16-9273-2016.

Riechelmann, T., Y. Noh, and S. Raasch, 2012: A new method for large-eddy simulations of clouds with Lagrangian droplets including the effects of turbulent collision. New J. Phys., 14, 065008), https://doi.org/10.1088/1367-2630/14/6/065008.

Rogers, R. R., and M. K. Yau, 1989: A Short Course in Cloud Physics. Pergamon Press, 293 pp.

Rothermel, J., and E. M. Agee, 1980: Aircraft investigation of mesoscale cellular convection during AMTEX 75. J. Atmos. 
Sci., 37, 1027-1040, https://doi.org/10.1175/1520-0469(1980) 037<1027:AIOMCC >2.0.CO;2.

Sagaut, P., 2006: Large Eddy Simulation for Incompressible Flows. Springer, $556 \mathrm{pp}$.

Sato, Y., S.-i. Shima, and H. Tomita, 2018: Numerical convergence of shallow convection cloud field simulations: Comparison between double-moment Eulerian and particle-based Lagrangian microphysics coupled to the same dynamical core. J. Adv. Model. Earth Syst., 10, 1495-1512, https://doi.org/10.1029/2018MS001285.

Shaw, R. A., W. C. Reade, L. R. Collins, and J. Verlinde, 1999: Reply. J. Atmos. Sci., 56, 1437-1441, https://doi.org/10.1175/ 1520-0469(1999)056<1437:R>2.0.CO;2.

Shima, S.-I., K. Kusano, A. Kawano, T. Sugiyama, and S. Kawahara, 2009: The super-droplet method for the numerical simulation of clouds and precipitation: A particle-based and probabilistic microphysics model coupled with a non-hydrostatic model. Quart. J. Roy. Meteor. Soc., 135, 1307-1320, https:// doi.org/10.1002/qj.441.

Shipway, B., and A. Hill, 2012: Diagnosis of systematic differences between multiple parametrizations of warm rain microphysics using a kinematic framework. Quart. J. Roy. Meteor. Soc., 138, 2196-2211, https://doi.org/10.1002/qj.1913.

Sölch, I., and B. Kärcher, 2010: A large-eddy model for cirrus clouds with explicit aerosol and ice microphysics and Lagrangian ice particle tracking. Quart. J. Roy. Meteor. Soc., 136, 2074 2093, https://doi.org/10.1002/qj.689.

Squires, P., 1952: The growth of cloud drops by condensation. I. General characteristics. Aust. J. Sci. Res., 5, 59-86.

Stevens, B., and Coauthors, 2003: Dynamics and Chemistry of Marine Stratocumulus-DYCOMS-II. Bull. Amer. Meteor. Soc., 84, 579-594, https://doi.org/10.1175/BAMS-84-5-579.

_ tions via observations of nocturnal marine stratocumulus. Mon. Wea. Rev., 133, 1443-1462, https://doi.org/10.1175/ MWR2930.1.
Stevens, D., and C. Bretherton, 1999: Effects of resolution on the simulation of stratocumulus entrainment. Quart. J. Roy. Meteor. Soc., 125, 425-439, https://doi.org/10.1002/ qj.49712555403.

Su, C.-W., S. K. Krueger, P. A. McMurtry, and P. H. Austin, 1998: Linear eddy modeling of droplet spectral evolution during entrainment and mixing in cumulus clouds. Atmos. Res., 4748, 41-58, https://doi.org/10.1016/S0169-8095(98)00039-8.

Tölle, M. H., and S. K. Krueger, 2014: Effects of entrainment and mixing on droplet size distributions in warm cumulus clouds. J. Adv. Model. Earth Syst., 6, 281-299, https://doi.org/10.1002/ 2012MS000209.

Vaillancourt, P., M. Yau, P. Bartello, and W. W. Grabowski, 2002: Microscopic approach to cloud droplet growth by condensation. Part II: Turbulence, clustering, and condensational growth. J. Atmos. Sci., 59, 3421-3435, https://doi.org/10.1175/ 1520-0469(2002)059<3421:MATCDG>2.0.CO;2.

Wang, S., Q. Wang, and G. Feingold, 2003: Turbulence, condensation, and liquid water transport in numerically simulated nonprecipitating stratocumulus clouds. J. Atmos. Sci., 60, 262278, https://doi.org/10.1175/1520-0469(2003)060<0262:TCALWT> 2.0.CO;2.

Wood, R., 2012: Stratocumulus clouds. Mon. Wea. Rev., 140, 23732423, https://doi.org/10.1175/MWR-D-11-00121.1.

Yamaguchi, T., and D. A. Randall, 2008: Large-eddy simulation of evaporatively driven entrainment in cloud-topped mixed layers. J. Atmos. Sci., 65, 1481-1504, https://doi.org/10.1175/ 2007JAS2438.1.

- , and - 2012: Cooling of entrained parcels in a large-eddy simulation. J. Atmos. Sci., 69, 1118-1136, https://doi.org/ 10.1175/JAS-D-11-080.1.

_ , and G. Feingold, 2013: On the size distribution of cloud holes in stratocumulus and their relationship to cloud-top entrainment. Geophys. Res. Lett., 40, 2450-2454, https://doi.org/ 10.1002/grl.50442. 